\title{
Near-Field Flow of Submarines and Ships Advancing in a Stable Stratified Fluid
}

\author{
Mehdi Esmaeilpour, J. Ezequiel Martin, Pablo M. Carrica* \\ Department of Mechanical and Industrial Engineering and IIHR-Hydroscience and Engineering, \\ The University of Iowa, Iowa City, IA 52242, USA \\ *Corresponding author, pablo-carrica@uiowa.edu
}

\begin{abstract}
A methodology is presented to predict density stratified flows in the near-field of naval vessels. The approach uses a single-phase level set method for the free surface, a dynamic overset technique to handle motions and controllers for self-propulsion and maneuvering. The density is solved with a higher-order transport equation coupled with momentum and mass conservation. Turbulence is implemented with a $k-\varepsilon / k-\omega$ based Delayed Detached Eddy Simulation (DDES) approach modified to add density gradients. Evaluation tests were performed for a two-dimensional square cavity, including grid and time step studies, and the stratified flow past a sphere, showing good agreement with available data. The stratified flow was studied for a self-propelled ship and a submarine. Density, velocity, pressure and turbulent quantities at the exit plane of the near-field contain a description of the relevant scales of the flow and can be used to compute the far-field stratified flow. It is shown that, as is the case of surface waves, the generation of internal waves requires energy that results in an increase in resistance. Moreover, the presence of a density interface against the hull results in a thickening of the boundary layer, just as in a solid/free surface juncture flow.
\end{abstract}

Keywords: Stratified flow; Near-field wake; Internal waves; Computational ship hydrodynamics. 


\section{Introduction}

Stratification in the ocean occurs as a result of changes in salinity, temperature or sediment concentration, leading to horizontal or vertical fluid density variations. Just as surface waves can appear at the interface between two fluids, sustained periodic motions known as internal waves can appear under disturbances on stable stratified conditions. Stable stratification in the ocean can be disrupted by different natural processes including breaking waves, wind, Coriolis forces and tides, resulting in flows of interest in oceanography; the passage of a body, such as a surface ship or a submarine, can have a similar effect. The study of stratified flow problems is strongly scale-dependent: for the current application of flow in the near-field of a ship (defined as the region immediately close to the vessel, where all flow scales down to the boundary layer scales are relevant), perturbations to a horizontally uniform, stable vertical density profile will be considered.

The motion of ships and submarines in stratified media is a problem of importance and the focus of this paper. A surface ship or an underwater vehicle advancing in a stratified fluid generates complex flow structures, which in the far-field are characterized by internal waves in the bulk flow. These internal waves can be used to detect vessels: for instance, they can be observed using remote sensing devices (Watson et al., 1992; Jackson et al., 2013). Features of the oceanic stratification, such as the presence of a strong pycnocline (defined as a region where the density changes rapidly with depth separating the well mixed surface layer from the denser deeper layer), can also be used to avoid detection, and for this reason submarines often operate below the pycnocline surface, which can disrupt the acoustic signals of sonars. While the effects of stratification on resistance and propulsion can be strong for extreme conditions, as is the case of dead-water (Miloh et al., 1993), the main focus of the present study of stratification is the generation of wakes and signature effects. There is a considerable difference in scales between 
the near-field flow, which is restricted to the region around the vessel, and the far-field flow, where the internal waves are important at normal ship operational conditions. The near-field flow is produced by the direct interaction of the stable stratified flow in the ocean with the advancing vessel, while the far-field is the evolution of the downstream signature of that interaction. To a very good approximation the near-field and far-field computations can be decoupled, since the far-field flow has little effect on the near-field and can use information from the near-field calculation as input to predict the evolution of the internal waves away from the vessel. This paper focuses on a methodology to predict the near-field density stratified flows of ships and submarines, and the study of such flows for self-propelled vessels.

Available numerical and experimental studies for interaction of bodies and stratified fluids have focused on simple geometries such as spheres and cylinders at low and moderate Reynolds numbers typical of a laboratory setup. Some of these results will be discussed in the following sections as evaluation tests for the present implementation of the computational fluid dynamics (CFD) solver. In the following paragraphs a brief description of the available data is presented.

Boyer et al. (1989) investigated the flow of a linearly stratified fluid past a long circular cylinder in a channel through a series of experiments. Results were presented for various Reynolds $(R e)$ and internal Froude $(F i)$ numbers and fluid depth ratios covering a wide range of flow regimes. Note that the internal Froude number, which is relevant for internal waves, relates to the standard free surface wave Froude number by $F i=F r \sqrt{\frac{\rho_{0}}{\Delta \rho}}$, with $F r=U_{0} / \sqrt{g L_{0}}$. A similar experimental study was conducted by Lin et al. (1992a,b) for the stratified flow past a sphere and different flow patterns were mapped on a detailed $R e$ versus $F i$ flow regime diagram. Bonneton et al. (1993) presented an experimental investigation related to the internal gravity wave field generated by a sphere towed in a stratified fluid. A fluorescent dye technique was 
used to show the difference between lee waves generated by internal waves and random waves generated by turbulence from the wake. Spedding et al. (1996) showed the complex effects of stratification on the far-field wake of a sphere, observing that even though the vertical velocity is much smaller than the horizontal one, the wake scaling corresponds better to an axisymmetric, unstratified wake than to a planar wake. Meunier and Spedding (2004) examined the effect of body geometry on the intermediate and late wake structure in a stratified fluid at moderately high Fi by comparing the wakes of various bluff bodies. It was observed that the size and amplitude of the long-lasting wakes strongly depend on the body geometry. Meunier and Spedding (2006) investigated the wake of a bluff body in three configurations: momentum-excess, zeromomentum, and zero-momentum with a slight angle of attack, finding that the coherent structures in the wake were different for all three cases. Brucker and Sarkar (2010) used direct numerical simulation (DNS) to study towed sphere and self-propelled spheroid wakes at $R e=50,000$. The authors compared the mean velocity and observed that in the self-propelled case, due to the higher shear, the mean velocity decayed more rapidly than for the towed case, resulting in a faster rate of energy transfer to turbulence. Using the same simulation, de Stadler et al. (2010) studied the turbulent wake behind an accelerating self-propelled axisymmetric body in a stratified environment. To create the acceleration for studying the effects of excess momentum on the initially momentumless self-propelled wake, a velocity profile corresponding to net thrust was added to the self-propelled velocity which resulted in small and moderate excess momentums. The study shows that parameters such as wake width, mean kinetic energy and defect velocity depend on amount and shape of this excess momentum. Also, the turbulent kinetic energy (TKE) and the dissipation rate increase as the shear in the mean profile increases.

Some efforts studying the effects of density stratification on surface ship waves are available in the literature; in general these efforts are geared towards a description of the far-field 
rather than the accurate prediction of the interaction between the ship and the stratification field. Earlier works by Hudimac (1961) and Crapper (1967) presented analytical approaches to study the internal wave modes caused by a moving body in a two-layered ocean. It follows from their work that, just as for surface waves, at ship speeds sufficiently larger than the internal wave speed only divergent waves travel downstream of the ship, while both divergent and transverse waves are present for slower vessels. Tulin et al. (2000) suggested a nonlinear theory to capture internal wave behavior at high $F i$ in weakly stratified flow that compared satisfactorily with available experimental results for a semi-submerged spheroid. Chang et al. (2006) presented one of the few available examples of CFD computation for a vessel in a stratified medium. They computed the generated internal and surface waves of the notional DARPA Suboff submarine advancing in a two-layer fluid, for different Froude numbers, and found the evolution of the wave pattern consistent with previous predictions.

In this work the evolution of the stratified flow in the near-field of a surface ship and a submarine is studied. The description of the vessels' geometry includes their moving control surfaces and propellers, resulting in localized intense mixing absent in previous calculations of this type of problem. Specific implementations to the CFD code to address variable density effects in momentum transport and turbulence modeling are discussed. Evaluation of the implementation is discussed for a two-dimensional square cavity and the three-dimensional stratified flow past a sphere. Finally, demonstration cases for the surface ship Athena R/V and the notional submarine Joubert operating near the surface in a stably stratified fluid are presented. 


\section{Modeling}

Variable density capabilities were incorporated to REX, a URANS code with DES/DDES capabilities, specifically designed for naval applications. As opposed to the more limited Boussinesq approximation, where body forces caused by density variations are accounted for but all other effects of density are neglected, variable density effects are fully incorporated into the conservation equations. The incompressibility assumption for the fluid is preserved since density changes are only due to external parameters (temperature, salinity, sediments) and not to pressure. The implementation also includes modifications to the turbulence model to take into account the effects of stratification on TKE production. The model consists of a scalar transport equation for the density, changes in the definition of the piezometric pressure to maintain a zero piezometric pressure in the far field and free surface, and modifications to the turbulence model to account for turbulence generation or suppression by density gradients. For density changes caused by variations in salinity or temperature, in which the water remains incompressible (meaning that pressure changes do not affect density), most terms needed are accounted for, including density changes in the momentum and continuity equations, but at this time the viscosity is still considered constant. Future work will include viscosity change effects too, though viscosity changes with the salinity and temperature variations in the ocean are small, and for most applications can be neglected. The scalar transport is standard and follows the same numerical methodologies as the momentum transport, and the turbulence treatment is taken from Yeoh and Tu (2010) and Venayagamoorthy et al. (2003).

A brief summary of the governing equations and their implementation is presented next.

\subsection{Governing Equations}


The dimensionless Navier-Stokes conservation equations governing the stratified flow are expressed as:

$$
\begin{aligned}
& \frac{\partial}{\partial \mathrm{t}}\left(\tilde{\rho} R i F r^{2}\right)+\nabla \cdot\left(\left(1+\tilde{\rho} R i F r^{2}\right) \mathbf{u}\right)=0 \\
& \begin{aligned}
\frac{\partial}{\partial \mathrm{t}}\left(\left(1+\tilde{\rho} R i F r^{2}\right) \mathbf{u}\right)+\nabla \cdot\left(\left(1+\tilde{\rho} R i F r^{2}\right) \mathbf{u} \otimes \mathbf{u}\right) \\
=-\nabla \hat{\mathrm{p}}+\nabla \cdot\left(2\left(\frac{1}{R e}+v_{\mathrm{t}}\right) \nabla^{\mathrm{s}} \mathbf{u}\right)-R i \rho^{*} \mathbf{k}
\end{aligned}
\end{aligned}
$$

where $t$ is the dimensionless time, $\mathbf{u}$ is the dimensionless velocity, $v_{\mathrm{t}}$ is the turbulent viscosity as prescribed by the turbulence model, and $\nabla^{S} \mathbf{u}=\left(\nabla \mathbf{u}+\nabla^{T} \mathbf{u}\right) / 2$ is the symmetric part of the velocity gradient. $\tilde{\rho}=\left(\rho-\rho_{0}\right) / \Delta \rho$ and $\rho^{*}=\left(\rho-\rho_{H}(z)\right) / \Delta \rho$ are dimensionless excess densities with respect to the reference density $\rho_{0}$ or the background stratification $\rho_{H}(z)$, with $\Delta \rho$ a reference density difference, typically chosen as the density difference from the free surface to a reference depth for which changes in density are negligible during the evolution of the flow. $R e=U_{0} L_{0} / v$ is the Reynolds number and $R i=\frac{\Delta \rho}{\rho_{0}} \frac{g L_{0}}{U_{0}^{2}}$ is the bulk Richardson number, with $v$ the fluid viscosity, $g$ the acceleration due to gravity; $U_{0}$ and $L_{0}$, are the reference velocity and length. The Richardson number can also be expressed as an internal, reduced or densimetric Froude number, $F i$, since $R i=\frac{\Delta \rho}{\rho_{0}} \frac{g L_{0}}{U_{0}^{2}}=\frac{\Delta \rho}{\rho_{0}} \frac{1}{F r^{2}}=\frac{1}{F i^{2}}$, with $F r=U_{0} / \sqrt{g L_{0}}$ the usual (free surface) Froude number. The dimensionless piezometric pressure $\hat{\mathrm{p}}$ is defined by $\hat{\mathrm{p}}=\frac{\mathrm{p}}{\rho_{0} U_{0}^{2}}+\frac{2}{3} k+R i \int_{0}^{z}\left(\frac{\rho_{H}\left(z^{\prime}\right)}{\Delta \rho}\right) d z^{\prime}$, with $\mathrm{p}$ the absolute pressure and $k$ the turbulent kinetic energy. The hydrostatic contribution to the piezometric pressure from the variable density profile is such that, under unperturbed conditions, the body force in Eq. 2 vanishes. This choice contributes to the numerical stability of the resulting scheme, and facilitates the implementation of Dirichlet boundary conditions for the piezometric pressure field at inlets and far-field conditions, as they reduce to the standard uniform density case $(\hat{p}=0)$. The choice of reference 
length for the Richardson number is the ship length $L_{0}$, as typically used for ship hydrodynamics, but it is less representative of the stratification characteristics, for which a representative depth would be preferable.

A standard scalar transport equation for the dimensionless density is implemented as:

$\frac{\partial}{\partial t}(\tilde{\rho})+\nabla \cdot(\mathbf{u} \tilde{\rho})=\nabla \cdot\left[\left(\frac{1}{R e \cdot P r}+\frac{v_{\mathrm{t}}}{P r_{t}}\right) \nabla \tilde{\rho}\right]$

where $\operatorname{Pr}=v / \alpha$ is the Prandtl number with $\alpha$ the molecular diffusivity, and $\operatorname{Pr}_{t}$ is a turbulent Prandtl number. The current implementation does not differentiate the possible sources of density variability (temperature, salinity, etc.) and uniform Prandtl value is used; moreover, strong turbulent mixing is assumed, under large Reynolds number conditions, which yields $\frac{1}{\operatorname{Re} \cdot \operatorname{Pr}} \ll \frac{v_{\mathrm{t}}}{P r_{t}}$ and $P r_{t} \approx 1$, implying that the transport of the contaminant causing the density difference is independent of its molecular properties (Shih et al., 2005). This is not necessarily true at the smallest flow scales that are encountered in a DNS or even an LES simulation, but it is a good approximation for a RANS solver. Also, it might not be a good approximation for the farfield wake, as Reynolds numbers decrease and molecular diffusivity effects become more important. While not implemented yet, a multi-species scheme that can account for the separate transport of different contaminants can be easily formulated if needed.

A level set function $\phi$ is used to calculate the position of the free surface:

$\frac{\partial \phi}{\partial \mathrm{t}}+\mathbf{u} \cdot \nabla \phi=0$

The level set function represents the distance to the free surface, positive in water. The solution algorithm requires a proper reinitialization process to accurately model surface waves; details are discussed in Carrica et al. (2007a). 
The blended $k-\varepsilon / k-\omega$ model (Menter, 1994) already implemented in REX, has been modified to include a buoyancy source term (Venayagamoorthy et al., 2003; Yeoh and Tu, 2010):

$$
\begin{gathered}
\frac{D k}{D t}=2 v_{t} \nabla^{s} \mathbf{u}: \nabla^{s} \mathbf{u}+\nabla \cdot\left[\frac{1}{P e_{k}} \nabla \mathrm{k}\right]-\beta k \omega+R i \frac{v_{t}}{\sigma_{\rho}} \frac{\partial \tilde{\rho}}{\partial z} \\
\frac{D \omega}{D t}=2 \gamma \frac{\omega}{k} v_{t} \nabla^{s} \mathbf{u}: \nabla^{s} \mathbf{u}+\nabla \cdot\left[\frac{1}{P e_{\omega}} \nabla \omega\right]-\beta \omega^{2}+2\left(1-F_{1}\right) \frac{\sigma_{\omega 2}}{\omega} \nabla \omega . \nabla k \\
+\alpha_{3} R i \frac{v_{t}}{\sigma_{\rho}} \frac{\omega}{k} \frac{\partial \tilde{\rho}}{\partial z}
\end{gathered}
$$

where $F_{1}$ is a blending function that transitions the solution between the two models $(k-\omega$ is used near the wall, while the less dissipative $k-\varepsilon$ is used in the free stream). The turbulent Peclet numbers for $k$ and $\omega$ are defined as:

$P e_{k}=\frac{1}{1 / R e+\sigma_{k} v_{t}} \quad, \quad P e_{\omega}=\frac{1}{1 / R e+\sigma_{\omega} \nu_{t}}$

The equation constants can be found in Menter (1994). The buoyancy terms in Eqs. (5) and (6) are modeled following Yeoh and Tu (2010) with:

$\alpha_{3}=F_{1} \alpha_{1}+\left(1-F_{1}\right) \alpha_{2}$

where $\alpha_{1}=0.31$ and $\alpha_{2}=0.44$. Also a turbulent Schmidt number $\sigma_{\rho}=1$ is defined for the buoyancy terms. The previously implemented DDES approach based on the $k-\varepsilon / k-\omega$ model is used without additional modifications (see Mofidi and Carrica, 2014 and references therein for details). A simulation using the original RANS $k-\varepsilon / k-\omega$ formulation was also performed. The model of Venayagamoorthy et al. (2003) has been tested in a variety of stratified flows, among others by Serchi et al. (2012) for density currents, and Ezz and Imran (2014) for submarine channels.

\subsection{Numerical methods}


The described methods have been implemented in REX, a CFD solver based on structured grids using overset technology to provide dynamic connectivity between the different grids (Carrica et al., 2007a). Suggar (Noack 2005) or Suggar++ (Noack et al., 2009) are used to compute connectivity interpolations, in different configurations, including implicit and explicit approaches. Body fitted grids allow for accurate geometric representation of any object present in the domain. Full six-degrees-of-freedom (6DoF) computations for objects are available, geared toward naval applications such as maneuvering and seakeeping. A multi-body hierarchy is used to include realistic motions of appendages and propulsors of parent bodies (Carrica et al., 2007b). Propulsion models (Chase et al., 2013; Martin et al., 2015) are available as an option to the direct simulation of a rotating discretized propeller. The fluid solver of REX is based on higher (up to fourth order) finite differences discretization of the presented equations and ADI solvers, and uses a projection method for strong pressure-velocity coupling. The code is fully parallelized for efficient calculation of large problems (previous calculations of up to 300 million grid points have been performed), with on-going work to extend capabilities to larger motions problems. Further implementation and numerical details regarding the solver can be found in $\mathrm{Li}$ et al. (2015) and references therein.

For the current simulations, the time derivatives in momentum and density equations are discretized using a second order Euler backward difference scheme. Convection terms in the density and momentum equations are discretized with higher-order upwind schemes, either second order full upwind for RANS or fourth order upwind biased for DDES. Non-linear, total variation diminishing (TVD) methods, including the implementation of flux limiters, previously available in the solver for transport of other quantities (Ismail et al., 2010) can also be used for density simulation. These methods are particularly useful for the simulation of this variable, as they avoid unrealistic under- and overshoots of the solution. The projection method is employed 
to couple pressure and velocity effectively and the resulting linear system is solved using the PETSc (Balay et al., 2013) toolkit. In order to solve the system of discretized governing equations, between two and four inner iterations are used in each time step. This typically results in residuals dropping two orders of magnitude. Since the time step is small for these computations (see Sections 4.1 and 4.2), solutions changes between time steps are moderate, supporting use of a small number of inner iterations.

Solution of variable density problems can produce robustness and stability problems. The pressure/velocity coupling methodology for variable density problems discussed in Li et al. (2015) in the context of high void fraction bubbly flows is used also herein. Density changes due to salinity or temperature in the ocean are mild and rarely exceed $3 \%$ and cause negligible effects in robustness. This small change in density does not require specific selection of numerical parameters as is the case for bubbly flows with high void fractions, which can result in local density ratios of 100 or more and require careful selection of relaxation parameters, time step, etc.

Boundary conditions for the density field are implemented in a similar fashion to all other transported quantities. Inlet profiles are currently implemented either as a high-order polynomial expression with dependency on the vertical coordinate or via linear interpolation from a provided look-up table. The integrated hydrostatic pressure associated with the background variable density, used for imposition of boundary conditions for pressure at the free-surface (Carrica et al., 2007a) and for the calculation of forces on solid surfaces of the moving bodies, is also presented as a look-up table, either pre-calculated at initialization or user provided. For all variables except the pressure, a zero gradient condition normal to the free surface is imposed using an extension transport equation in the air phase (Carrica et al., 2007a), while the dimensionless atmospheric pressure is imposed at the free surface. 


\section{Test cases}

\subsection{Square Cavity}

The first test case, used to check the implementation of the density equation, is the laminar flow inside a 2D square cavity with a temperature gradient, which causes a density change. The relation between density and temperature is defined as $\rho=\rho_{0}\left(1-\beta_{T}\left(T-T_{\text {cold }}\right)\right)$, where $\rho_{0}$ is a characteristic density, and $\beta_{T}$ is the volumetric expansion coefficient. This case was studied by Al-Amiri et al. (2007), with the boundary conditions shown in Fig. 1. The upper boundary moves with constant unit velocity while all other boundaries are at rest. The side walls are adiabatic (which translates in the present formulation to a zero gradient condition, $\partial \tilde{\rho} / \partial x=$ 0 ), while the upper and lower walls have Dirichlet boundary conditions $\tilde{\rho}=0$ and $\tilde{\rho}=1$, respectively. $\operatorname{Pr}=1, R e=500$, and $R i=4 \times 10^{-4}$ were used. All results are shown in dimensionless units.

To evaluate grid convergence, computations were performed on four non uniform grids with $41 \times 41,81 \times 81,161 \times 161$ and $321 \times 321$ grid points, named Very Coarse, Coarse, Medium and Fine grids. Time steps were varied between $\Delta t=0.02$ for the Very Coarse grid to $\Delta t=0.0025$ for the Fine grid, maintaining a constant Courant number.

Figure 2 shows the axial velocity at the vertical centerline of the cavity, which is in excellent agreement with the work of Al-Amiri et al. (2007). Since the solutions for the Medium and Fine grids are indistinguishable in the figure, they are shown as a single line. Axial velocity contours for the medium grid are also shown in Fig. 2. The density profile at mid-section shown in Fig. 3 exhibits again remarkable agreement compared with the results of Al-Amiri et al. (2007). In all cases solutions for different refinements converge well in grid. Isocontours of 
density for this case are also depicted in Fig. 3, showing good agreement with Fig. 5 in Al-Amiri et al. (2007), though in their figure the authors do not show the contour magnitude.

Time step convergence was analyzed for the medium grid, using time steps $\Delta t=$ $0.04,0.02,0.01$ and 0.005 and evaluating solutions at $t=10$. Grid and time step convergence were studied following the procedures described in Stern et al. (2001). Results for the maximum vertical velocity and density gradients at the top and bottom walls for steady state for the grid study and at $t=10$ for the time step study are shown in Tables 1 and 2 . For these studies a very large number of inner iterations was used so that the iterative error could be neglected. The grid study using the Very Coarse, Coarse and Medium grids showed that the solutions converge, but the Very Coarse grid is not in the asymptotic range, yielding unrealistically high order of accuracy $\mathrm{P}_{\mathrm{G}}$. Using the Coarse, Medium and Fine grids the study results in an order of accuracy close to second for all three variables evaluated, as expected since all equations are discretized to second order in space except the convection terms which are fourth order. The uncertainty $\mathrm{U}_{\mathrm{GC}}$ is highest for the density gradient at the top wall at approximately $0.4 \%$ of the fine mesh value. The time step analysis using the three smallest time steps, shown in Table 2, yields an order of accuracy a bit lower than second, though in time all equations are discretized using a second order Euler backward scheme. This may be because the differences between time steps are very small (on the fifth or sixth significant digit) and even tighter iterative convergence may be needed. Using the three larger time steps the order of accuracy is slightly closer to second. In both cases the time step uncertainty $\mathrm{U}_{\mathrm{TC}}$ is very low, with the highest about $0.0006 \%$ in the bottom wall density gradient.

\subsection{Towed sphere}


Bonneton et al. (1993) conducted an experimental study on the generation of lee waves and wake-emitted internal waves. For a range of Reynolds and Froude numbers, and using visualization techniques appropriate to the study of internal waves, they observed and quantified the different lee and random waves generated in the wake of a sphere. In the experiment, spheres of different diameters were towed in a tank filled with a linear stable stratification of salty water. The buoyancy or Brunt-Väisälä frequency, defined by $N^{2}=-g / \rho_{0} d \rho / d z$, was used to characterize the flow conditions. For a given stratification and a given sphere size, as the towed velocity changes, the two dimensionless numbers $F i$ and $R e$ change together with the linear relation $\operatorname{Re}(F i)=\operatorname{Re}(1) F i$, where $\operatorname{Re}(1)=2 R^{2} N / v$ is the Reynolds number when $F i=1$. A constant $\operatorname{Re}(1)=253$, corresponding to experimental $N \approx 1.17 \mathrm{rad} / \mathrm{s}$ and $R=0.0112 \mathrm{~m}$, was used for all simulations, although experimentally $R e(1)$ was varied in a larger range by changing the sphere diameter. This $\operatorname{Re}(1)$ was chosen because of the extensive data available, and because is the lowest Reynolds number tested that allows computations in laminar mode without turbulence model.

Details of the overset grid system are shown in Fig. 4. The domain extends $50 D \times 20 D \times$ $20 D$ and is discretized in $327 \times 269 \times 269$ points in $x, y$, and $z$ directions, respectively, for a total of 23.7 M grid points. The grid for the sphere is discretized in three overset blocks for a total of $2.8 \mathrm{M}$ points. No turbulence model was used for the simulations.

Figure 5 shows experimental and linear theory results from Bonneton et al. (1993) along with the present CFD results of the wave field for $N t=0$ and $N t=11.7$, at the strongest stratification considered $F i=10 / \pi$, with $N t$ the time non-dimensionalized with the BruntVäisälä frequency $N$. The Reynolds number for this case is $\operatorname{Re}(1)=253(\operatorname{Re} \approx 805)$. The vertical displacement $\zeta$ of the lee wave in a horizontal plane is presented at $|z|=3 R$ below the center of the sphere. The lee wave pattern is clearly captured by the CFD simulation, and the 
wave amplitude and the angle between the phase line and the $x$-axis are in good agreement with the experimental and linear theory results. The same plane is shown in Fig. 6 for different internal Froude numbers, while maintaining the same $\operatorname{Re}(1)=253$ (as previously discussed, the actual Reynolds number increases linearly with $F i$ ). The results are consistent with Bonneton et al. (1993) observation of a change from lee-wave-dominated regime to a random-wavedominated one at $F i \approx 4.5$. Their observation of Reynolds-independent transition was not checked in this work, as a single $\operatorname{Re}(1)$ was considered.

The wavelength (Fig. 7) and wave amplitude (Fig. 8) were also analyzed. Notice that the experimental data by Bonneton et al. (1993) was measured at $\operatorname{Re}(1)=2614$, too high for a laminar simulation but too low for the high-Reynolds number turbulence model in REX. Random wavelength for the highest $F i$ number is not reported as it cannot be accurately obtained from our solution. Lee wave amplitude is also reported for the lowest $F i$ number only, as the random wave dominates over lee waves as $F i$ increases. The regression line in Fig. 7 shows the theoretical wavelength decrease as $1 / N t$ for random waves emitted by a local impulse (Bonneton et al., 1993). While there is some discrepancy between the computations and experimental results in Bonneton et al. (1993), likely due to the different Reynolds numbers used, Figs. 5 through 8 show a remarkable level of agreement between CFD and experiments, suggesting that the modeling approximations and numerical implementation are appropriate to capture the main features of the flow, including wavelength and amplitude of the internal wave in a stratified flow. 


\section{Naval hydrodynamics applications}

\subsection{Near-field stratified flow for a surface ship}

Computations were performed for the US Navy research vessel Athena $\mathrm{R} / \mathrm{V}$ to demonstrate the capabilities of the solver under stratified flow conditions and realistic ship geometry. Athena R/V is a decommissioned PG-94 Asheville-class patrol gunboat transformed into a high-speed research vessel in 1976; its main properties are presented in Table 3. Propulsion is provided by twin propellers of variable pitch rotating outward on top, powered either by two independent diesel engines for speeds up to 14 knots, or by a gas turbine that allows the vessel to reach a maximum speed of 35 knots. Self-propulsion computations are performed using discretized rotating propeller controlled by a PID speed controller, while the rudders are kept straight. The overset grid topology is shown in Fig. 9. The grid contains about 31 million grid points, distributed for parallel computation into 298 processors. The ship's grids are allowed to move in surge, heave and pitch, following the predictions of the motion solver, and restricted from sway, roll or yaw motions. Free surface refining grids only move with the ship in the horizontal plane. The propeller grids rotate around their shaft axis as prescribed by the speed controller, in addition to the vessel motions, using a hierarchical parent/children structure. The overset domain connectivity solver Suggar++ (Noack et al., 2009) was used, running in multiple lagged mode with 4 processes to match the execution time of the CFD solver. The background grid is Cartesian to more accurately impose the far-field boundary conditions.

The simulations are conducted for a fairly strong stratified condition with density profile obtained at the Kitimat Bay (BC) station as presented in Fig. 10. The data includes salinity, temperature and depth, and corresponds to a seasonal average based on weekly measurements (Fissel et al., 2010). The density distribution is approximated by piecewise polynomial functions 
on depth, thus the non-dimensional density profile is imposed as initial and inlet boundary conditions using an analytical expression. The bulk Richardson number is $R i=0.036$ and the Prandtl number was assumed $\operatorname{Pr}=1$. The self-propulsion simulation was conducted using a speed controller (Carrica et al., 2010) to achieve $U_{0}=10.5$ knots, corresponding to $F r=0.252$ and $R e=2.53 \times 10^{8}$. Computations were performed at full scale using wall functions and both RANS and DDES approach based on the turbulence model discussed in Section 2.1, with grids designed for a range of dimensionless wall distance $y^{+}=30-300$. A posteriori check of $y^{+}$at self-propulsion for the DDES simulation shows a mean value of 41 and a standard deviation of 21 , with values below 30 concentrated in regions downstream of appendages or in the stern where recirculations are present and friction force is small or negligible. Linear iterations to solve each dimensionless variable are converged to a residual of $10^{-5}$, except the pressure which is converged to $10^{-6}$. Final self-propulsion conditions were obtained after $65 \mathrm{~s}$, with a time step of $2.18 \mathrm{~ms}$ (total 30000 steps), then three additional ship lengths were run to transport the density and to perform computations of averages and turbulence quantities. Simulations were also performed with a constant density of $1023.3 \mathrm{~kg} / \mathrm{m}^{3}$ (equivalent to $\rho_{0}+\Delta \rho$ ) and using the same density function as in stratified flow but as a passive scalar $(R i=0)$ to evaluate differences when sailing in stratified seas. Notice that a better choice for the density for the passive scalar computations could have been a number closer to $1000 \mathrm{~kg} / \mathrm{m}^{3}$, more in line with the density of the fluid where the ship is located in the Kitimat profile. However, this choice is of little relevance, since forces are only reported for a rough comparison, and the effect on the Reynolds number, which can affect vortical structures and mixing, is small. The ship effective power to overcome resistance is computed in CFD directly from pressure and viscous forces, resulting in $140.6 \mathrm{~kW}$ for the case with stratified flow and $144.1 \mathrm{~kW}$ for the case with constant density (140.8 $\mathrm{kW}$ if a density of $1000 \mathrm{~kg} / \mathrm{m}^{3}$ is used). Extrapolating to $10.5 \mathrm{knots}$ the powering data 
from Crook (1981) in model scale, who measured from 14 knots to 35 knots, results in $153 \mathrm{~kW}$ after correcting for the $5 \%$ difference in displacement between the experimental conditions and those of this paper. Given the different conditions this number can only be used as a rough check.

As the ship advances through a calm and stable stratified background ocean it disturbs the flow and creates unstable features due to stirring that propagates downstream. The wavelength of the internal wave that is generated is much longer than the ship length, suggesting that the nearship field and the far-field stratified flows can be computed separately, with the more expensive near-field simulation limited to about one ship length and the far-field using the exit of the nearfield as inlet condition. Performing a fully resolved simulation of the near- and far-fields together would be extremely expensive due to the large size of the required domain, and the vastly different time step needed to model the rotating propeller, the fastest process in place, and the slow advance of the internal wave. The coupling of near- and far-field stratified flow solvers is a subject of research and is not discussed in this paper.

The resulting density distribution at planes $y / L=0$ and $y / L=-0.04$ (approximately the propeller shaft axis location) are presented in Fig. 11, which shows the different layers of stratification resulting from the interaction of the stable stratified background with the selfpropelled advancing ship. Figure 11 shows that the density disturbances on the near wake are related to separation at the wet transom and propeller mixing, and to a lesser extent to the free surface wave system caused by the ship. Both instantaneous and average results are shown for both turbulent models; as expected, flow structures are better captured by the DDES approach. Differences in the mean are only important in the wake, away from solid surfaces, and are related to a lower prediction of mixing by RANS modeling. The downstream evolution of the wake can be also appreciated in Fig. 12, which shows several longitudinal cross sectional planes colored 
with density and the free surface colored with axial velocity. Figure 13 shows the resolved and modeled TKE for the same planes as in Fig. 12. For comparison, the transport of a passive scalar with the same inlet distribution as the background density is also shown in Figs. 12 and 13. Notice that the resolved TKE is computed using point averages for all times, and not a phaseaveraged calculation with the propeller blade position, which would require very long statistics not available from the simulations. This results in reliable TKE results in the transom wake but results closer to the mean square of the fluctuations in the wake of the propellers. While details of the instantaneous flow differ, it is found that for the simulated conditions and for the near wake, stratification effects are small compared to inertial effects. As the wake velocity decays, most evident in the furthest downstream cross section in Fig. 12 at approximately one ship length from the stern, the strong stirring signature from the propellers that push light fluid down and pull heavy fluid up, and from the stern that pulls heavier fluid closer to the free surface, is preserved. The stratification volume forces tend to maintain the wake defect higher near the surface than in the non-stratified case with the passive scalar, and similarly a stronger propeller wake. At this plane, modeled TKE is weaker for the non-stratified case in the region immediately above the propeller wake, suggesting that the unstable stratification in that region is at this distance strong enough to have an effect as a sink of TKE. Electronic Annex I presents an animation of the self-propelled condition in stratified flow. Time has been slowed down to a fourth of the full scale value, to better show the variations of the density field in the wake. Notice the compression of the density isolines between the mixed region caused by the propeller action and the undisturbed background, as well as the slow pulsing of the region between the two propeller wakes under the action of the surface waves originated in the ship's transom. 
Note also that self-propelled at 10.5 knots the ship's wake is not momentumless due to wave-making resistance, resulting in net positive momentum of approximately $10 \%$ of the thrust in model scale in barehull condition (Jenkins, 1984).

\subsection{Near-field stratified flow for a submarine}

The second application case is the interaction of a submarine operating near the surface with a stratified flow. The submarine geometry corresponds to the Joubert BB2 model hull form with overall full scale length of $L_{0}=70.2 \mathrm{~m}$, which is a modified version of the original Joubert geometry designed by the Defence Science and Technology Organisation (DSTO) in Australia (Overpelt, 2015). The main particulars of Joubert BB2 and its propeller characteristics are given in Tables 4 and 5, respectively. The submarine is fitted with a MARIN stock propeller 7371R scaled up to full scale, with origin located $1.33 \mathrm{~m}$ forward of the aft perpendicular. The grid topology is shown in Fig.14. A Cartesian computational domain is used to impose the far-field boundary conditions. The overset grid system includes the hull, sail, propeller, stern planes, sail planes, refinements and domain background with 44.7 $\mathrm{M}$ or 48.7 $\mathrm{M}$ grid points, depending on the refinements used, distributed into up to 470 processors for the largest case. A longer background and refinement were used for one of the simulations to better capture the development of the internal wave. The simulations are conducted using a two-layer stratification represented by an hyperbolic tangent density distribution (see Fig. 10). Two stratification conditions are considered, one with the pycnocline located at the propeller centerline, and the second with the pycnocline located slightly below the submarine. A complementary simulation for the latter case considering the density distribution as a passive scalar is also presented. The bulk Richardson number based on the boat length is $R i=0.9$ for both stratified conditions; corresponding values based on depth are 0.225 and 0.297 , respectively. 
Self-propulsion simulations with discretized propeller at full scale are conducted in calm water to achieve a speed of 10 knots, corresponding to $F r=0.195$ and $R e=3.6 \times 10^{8}$. The depth was set at $0.25 L_{0}$, so that the propeller shaft is located $17.55 \mathrm{~m}$ below the undisturbed free surface. Motions of the vessel were restricted on all directions except surge. This simplification was adopted to isolate the effect of propulsion on the stratified fluid; in the future full six-degrees-of-freedom might be included to study effect of stratification in steady roll angle and pitch, though the final attitude at self-propulsion in experiments shows very small pitching angle and stern plane deflection (Overpelt, 2015). Self-propulsion is achieved after approximately $140 \mathrm{~s}$ with a time step of $4.1 \mathrm{~ms}$ (about 34,000 time steps); simulations were then run for a few additional ship lengths at self-propulsion to obtain averaged quantities. The turbulence was modeled for all cases using DDES; an a posteriori check of the dimensionless wall distance shows that at self-propulsion the average distance is $y^{+}=50$, with a standard deviation of 27.6. About $10 \%$ of the active surface points are below the recommended range of 30-300 for the application of the model; these points occur in areas of particularly low shear on the hull, near the propeller, where the friction forces are very small.

Animations of the cases studied are shown in Electronic Annexes II, III and IV. Electronic Annex II shows the internal interface in the near wake of the Joubert BB2 submarine with the pycnocline located at $z_{0}=-0.33 L_{0}$, for the cases stratification and with a passive scalar. Electronic Annex III compares the near wake for pycnocline located at the shaft depth $z_{0}=-0.25 L_{0}$ and the deeper pycnocline shown in Electronic Annex II at $z_{0}=-0.33 L_{0}$. Electronic Annex IV exhibits a zoomed out view that better shows the development of the internal waves.

Figures 15 and 16 show instantaneous results for the different self-propulsion cases simulated. In Fig. 15 the two stratified cases considered are shown. The distortion of the density 
profile as result of the direct mixing and stirring due to the propeller action is evident on the case with the pycnocline situated at the propeller centerline. Notice that the profile downstream of the propeller is sharper than the original profile, due to the concentration of streamlines in the converging flow in the stern of the hull, that constitutes the incoming flow into the propeller plane (see inset in Fig. 15). Also, much finer grids are used to resolve the propeller and wake flows, thus maintaining the sharper profile, at least until it emerges into the coarser background grid. In the second case, changes to the density profile occur indirectly, as fluid acceleration near the submarine creates an internal wave at the interface, see also Electronic Annex III. No appreciable difference is observed on the free surface waves for the two cases, which are mainly the response to the submarine's pressure field as it sails near the surface. Variations on the vortical structure of the propeller and submarine's stern planes are better observed in Fig.16 (insets). Vortices evolving near the pycnocline are noticeably modified by its presence, most notably the propeller's tip vortices in the case $z_{0}=-0.25 L_{0}$, and the lower stern planes' tip vortices for $z_{0}=-0.33 L_{0}$. Vortices away from regions with varying density resemble those for the passive scalar case (propeller tip vortices for $z_{0}=-0.33 L_{0}$; upper stern planes' tip vortices). Notice that in all cases the presence of the sail wake distorts the tip vortices in the upper region of the propeller wake; the presence of the stratified interface has an equivalent effect.

The generation of an internal wave can be observed in Fig. 16. For the reference, nonstratified case, the only effect observed is the deformation of the interface as the propeller wake diffuses and increases in size, but such deformation has no effect on the dynamics of the interface that can only be transported by the flow as a passive scalar, see also Electronic Annex II. For the other two cases (animations in Electronic Annexes III and IV), the presence of the submarine generates a standing internal wave, similar to those observed for the sphere case 
shown previously. It is interesting to note that the internal wave amplitude is much larger when the interface does not interact directly with the body, though the wavelength does not change. The two main reasons for this behavior are a) a reduction in stirring as the propeller does not interact with the interface, but more importantly b) the lack of a vertical acceleration of the fluid in the region between the submarine and the interface; instead only lateral perturbations to the interface are present when the submarine operates at the interface.

The celerity of a two-layered, deep internal wave is related to the celerity of surface wave by $C_{i} / C_{s}=\sqrt{\Delta \rho /\left(\rho_{1}+\rho_{2}\right)}$, so the traditional Froude number for an internal wave is $F r_{i}=$ $\operatorname{Fr} \sqrt{\left(\rho_{1}+\rho_{2}\right) / \Delta \rho} \cong \sqrt{2} F i$. This relation is not valid for more complex situations, like the case of Athena $\mathrm{R} / \mathrm{V}$ in a littoral stratification with a complex profile, but can be used for the case of Joubert, where a two-layered stratification is used. For the conditions of the paper, $F r_{i} \cong 1.5$, which can in principle be used to estimate the internal wave wavelength, which in this case is $\lambda_{i} \cong 14 L_{0}$, and compares with a free surface wavelength $\lambda \cong 0.24 L_{0}$. That is consistent with what is shown in Figs. 15 and 16, where one wavelength of the internal wave cannot be captured by the length of the domain, but the surface wave is resolved well.

Average velocity and TKE (both resolved and modeled) are shown in Fig. 17 for a plane $0.12 L_{0}$ downstream of the propeller, for the non-stratified case and for the case with $z_{0}=$ $-0.25 L_{0}$. The average was taken over 200 instantaneous solutions, over a time of about $8.2 \mathrm{~s}$ (about the time required to advance 0.6 ship lengths at self-propulsion). A fairly uniform sampling of blade positions was used to avoid locking blade structures on the average. All structures observed in Fig. 17 are truly part of the average field and not an artifact of the averaging process; these structure arise from heterogeneities of the incoming velocity profile due to the presence of low velocity regions in the wake of the control planes and the sail. Two important effects of the stratification are apparent on the mean flow: the presence of regions of 
reduced velocity immediately outside the tip vortex region and around the location of the density interface; and a flattening of the propeller wake in the vertical direction. This distortion of the wake does not result as a direct effect of stratification, as it occurs further downstream in regions where buoyancy forces dominate over inertial ones; but it occurs as a result of the reduction of streamwise velocity.

Velocity fluctuations are dominated by the propeller action and are fairly uniform inside the propeller disk wake, at about $10 \%$ the advance velocity; no strong difference is observed between the stratified and the non-stratified case. The modeled TKE is approximately two orders of magnitude smaller than the resolved portion, and clearly shows the effect of the velocity gradient near the density interface. Variations in TKE correlate well with the presence of stratification. Figure 18 shows the mean density and mean vertical density gradient, which contributes as a source (or sink, in the case of stable stratification) of the modeled turbulent quantities, as shown in Eqs. (5) and (6).

Figures 17 and 18 are useful to explain the variations observed in the wake of the propeller, based on the mean flow around it, but they are not sufficient to explain the origin of the low velocity region near the density interface. The boundary layer for the submarine is presented in Fig. 19, which shows that the origin of the low speed region at the interface is a thickening of the boundary layer as a result of up-swelling flow reaching the interface. This upflow reaches the interface and rises it, while at the same time the no penetration condition across the interface forces low velocity flow out of the boundary layer, in a similar fashion to what occurs in free surface flows where the boundary layer thickens at the free surface/hull interface (Longo et al., 1998). The boundary layer thickening occurs as the changes in pressure distribution due to the presence of the stratification affect the angle of attack of the submarine. This effect is clearly observed also by an increase in vorticity generated at the sail planes, far 
away from the stratification, that can only be explained by a change of direction of the incoming flow to the foil.

These changes in the flow pattern result in an increased resistance due to dynamic pressure for the submarine. Though it is not shown here, those changes are even more pronounced for the stratified case with the profile centerline below the submarine, as the direct action of the pressure field of the submarine results in larger interface deflection, and thus also in larger change in angle of attack for the incoming flow and a larger induced internal wave (see Fig. 16). To a great extent, as shown in the inset in Fig. 15, the density field follows the streamlines because the restoring gravitational forces are small in stratified flows. Thus an axisymmetric body in a two-layer stratified flow with a small density difference will cause little deflection of the interface if this interface is located exactly in the axis of revolution; in fact, if the density difference is infinitesimally small the interface is not deflected vertically at all, because streamlines incoming into the axisymmetric body in the plane of the interface remain in that plane. On the other hand streamlines close to the body and below are deflected down as they encounter the body, and thus the interface will do the same. There is a distance to the axis of revolution that produces the maximum deflection. Joubert is not a body of revolution, and thus it creates an internal wave for the case of the interface at the depth of the propeller shaft, but the internal wave is stronger when the interface is a bit deeper, and the resistance increases accordingly. As a result, the pressure coefficients for $z_{0}=-0.25 L_{0}$ and $z_{0}=-0.33 L_{0}$ increase to $1.2710^{-3}$ and $1.4510^{-3}$, respectively, from $1.2210^{-3}$ for the non-stratified case.

For all cases, changes in resistance due to friction are negligible compared to changes in pressure forces. The friction coefficients for $z_{0}=-0.25 L_{0}$ and $z_{0}=-0.33 L_{0}$ are $1.8410^{-3}$ and $1.8210^{-3}$, respectively, compared to $1.8310^{-3}$ for the non-stratified case. The pressure distribution will likely result in changes to the trim of the submarine for a fully free sailing case. 
That would have complicated the description of these striking effects of the stratification on the flow field, which is the reason for the current setup captive in all motions except surge. The energy implications of stratification are discussed in detail in the next section. 


\section{Propulsion and energy considerations}

The presence of a stratified background can affect the energy balance for a self-propelled vessel. Just as increased output from the propeller is needed to overcome the surface waves generated by a moving ship, any internal wave generated due to the presence of stratification incurs an energetic deficit in the ship system. The energy changes are however small, and can be masked by other changes in the ship configuration (such as change in draft and attitude) that are also a result of the presence of the stratified fluid. In this section a potential energy budget is proposed and results for the cases presented previously are discussed.

The potential energy available to generate internal waves is of interest. The rate of change of total potential energy in a control volume (or domain) of volume $V$ and surface boundaries $S$ can be computed as (Winters et al., 1995; Tseng and Ferziger, 2001):

$\frac{d E_{t o t}}{d t}=-\oint_{S} \rho g z \boldsymbol{u} \cdot \widehat{\boldsymbol{n}} d s+\int_{V} \rho w g d V+\oint_{S} \alpha g z \boldsymbol{\nabla} \rho \cdot \widehat{\boldsymbol{n}} d s-\alpha g\left(\bar{\rho}_{t o p}-\bar{\rho}_{\text {bottom }}\right) A$

where $z$ is the vertical spatial coordinate (positive upward). The first term on the right-hand side of Eq. (9) represents the change in potential energy due to the advective fluxes across the boundaries of the domain, the second term relates to exchange between potential and kinetic energy via buoyancy flux, the third term shows the change due to the diffusive mass flux across the boundaries, and the last term represents the change in potential energy based on mixing in the domain, with $A$ a horizontal cross sectional area where the densities at top and bottom are averaged. These last two terms are small for the near field and will be neglected. The third term exchanges energy within the domain and is thus not available for generation of internal waves in the far field. The potential energy leaving the exit boundary of the computational domain per unit time, represented by the first integral of the right-hand side of Eq. (9) over the exit surface of the domain $\left(x=x_{\max }\right)$, is then the potential energy available to generate internal waves in the far field, though part of this energy will be spent on turbulent and diffusive mixing. 
Equation (9) has the problem of including the free surface energy, which in the case of surface ships is typically much higher than the energy due to deviation from stable stratification. The energy leaving the exit plane per unit time, due only to deviation from stable stratification can alternatively be evaluated from

$$
P_{a v}=\oint_{A}-\left[\rho-\rho_{H}(\phi)\right]\left[\phi+z\left(\rho_{H}\right)\right] g \boldsymbol{u} \cdot \widehat{\boldsymbol{n}} d A=\oint_{A} I_{x} d A
$$

where the integrand explicitly evaluates the work needed to translate a particle of density $\rho$ with excess density $\rho-\rho(\phi)$ from its current depth $\phi$ respect to the free surface to its stable depth $-z(\rho) . \rho(\phi)$ is the stable equilibrium density stratification given in Fig. 10, and $z(\rho)$ is the depth at which a fluid particle of density $\rho$ is found in stable stratification, or essentially the inverse of the density distribution shown in Fig. 10. In Eq. (10) $I_{x}$ is the potential power flux crossing the plane due to deviations from stable stratification, referred here as potential power flux. Note that Eq. (10) measures the potential energy out of equilibrium, so an internal wave does have potential energy. These out of equilibrium density distributions are waves or can generate waves.

The proposed method was applied to self-propulsion results for Athena R/V. The total potential energy per unit time available for generation of internal waves, computed with Eq. (10), is estimated to be $2 \mathrm{~kW}$. Though this is a small fraction of the effective power, the small density difference on the stratified layers means that significant internal waves can still be produced. Note that part of this energy will be dissipated on turbulent density mixing. Calculations for Athena $\mathrm{R} / \mathrm{V}$ have the disadvantage of containing several competing processes affecting the outcome for a propulsion analysis, including uncertainty in the separation of potential energy from the internal and the surface wave, and different draft and attitude for the vessel for different density distributions. A further difficulty in assessing the effect of stratification on propulsion for 
this case is that the propellers operate at higher density (approximate $1005 \mathrm{~kg} / \mathrm{m}^{3}$, as shown in Fig.12) than the density of the fluid surrounding the hull which can improve propeller thrust, simply due to the increase of density, while any buoyant effects such as reduction of vertical mixing are negligible given the dominant effect of the propeller motion. Given that all these complexities, a complete energy budget study was not performed.

Simulations for the Joubert BB2 submarine, on the other hand, by restricting vertical motions of the vessel and using essentially a two layer system, allow for a clear separation of the generation of internal waves from other effects affecting the total resistance of the vessel. Table 6 presents results for the two stratified conditions (one with the propeller operating at the pycnocline, the second with the whole submarine above the interface), and their comparison to unstratified conditions. The effective power is defined as $P_{E}=R_{T} U_{0}$, with $U_{0}$ the self propulsion speed and $R_{T}$ the resistance, exactly balancing propeller thrust at self-propulsion. The shaft power is defined as $P_{p}=\omega Q$, with $\omega$ the propeller rotational speed in $\mathrm{rad} / \mathrm{s}$ and $Q$ the propeller torque. The extra power due to stratification reported in Table 6 is computed by subtracting the effective power in stratified flow from the effective power in the constant density flow. The potential energy flux for both stratified cases is much larger compared to the shaft power than it is for the previous case, and consistent with the increase in total resistance. The potential energy flux is about 5 times larger for the case with a deeper interface, as the initial internal wave setup is larger for that case, and mixing due to direct interaction of the pycnocline with the vessel is negligible. Figure 20 illustrates the distribution of $I_{x}$ in different longitudinal planes.

The thrust identity method (ITTC, 1978) was used to obtain the advance velocity and the propeller efficiency, based on the values for thrust from the simulation and the open water curve characteristics for propeller 7371R. The propeller efficiency is essentially unchanged with 
differences of about $0.1 \%$ across the different cases, while power varies as much as $8 \%$. The lack of strong dependence of propeller efficiency on stratified conditions supports the assumption that the stratification is too weak compared to the inertial forces resulting from the operation of the propeller to have a strong effect on the propeller dynamics.

Though the stratification and boat speed conditions evaluated herein are not as dramatic as those in a dead water situation, the trends show a considerable increase in torque when operating in strong stratification. Note that these computations are performed changing the propeller rotational speed to achieve self-propulsion. If the situation is such that the engine power is constant as the ship transitions from well mixed to stratified conditions, the increase in torque will result in a reduction in RPM and consequent slowdown of the vessel. 


\section{Conclusions}

A methodology to compute density stratified flows in the near-field of ship and submarine wakes is presented. The approach uses a density transport equation and modifications to the mass and momentum conservation equations and turbulence model equations to include appropriate effects due to changes in density. Boundary and initial conditions are also modified to impose a background density distribution. The methodology was implemented in the naval hydrodynamics code REX. Tests were performed for the cases of a heated square cavity, for which also grid and time step studies were performed, and a sphere advancing in a stably stratified fluid, showing in both cases good agreement with available experimental or numerical data.

Simulations were performed for the research vessel Athena $\mathrm{R} / \mathrm{V}$ and the notional Joubert BB2 submarine advancing in a stratified fluid. The computations were performed for a relatively strong stratification condition measured at the Kitimat Bay Station, in the case of Athena R/V, and for two-layer hyperbolic tangent profiles for Joubert BB2. Density disturbances on the near wake for both were analyzed.

It is observed that these near field wakes are mostly affected by two different processes for both vessels: the separation at the wet transom and propeller mixing in the case of Athena $\mathrm{R} / \mathrm{V}$, and the disturbance of the background density profile by the presence of the vessel for the case of the submarine. The generation of an internal wave for the surface ship case is not as evident from these simulations, as it is for the submarine. This is probably related to differences in stratification strength between the two cases, extension and discretization levels of wake refinements and vertical location of the vessel with respect to the density profile. While vertical position cannot be realistically changed for surface ships, the simulations presented for the 
submarine case show clearly that distance to the pycnocline strongly affect the internal wave generation due to the presence of the vessel.

The generation of the internal waves requires power that are reflected in an increase in resistance. This is shown clearly for the submarine, which was computed with the density change interface at two depths and also for a constant density. Moreover, if the interface is located deeper, immediately below the stern planes, produces a stronger internal wave and more resistance than when the interface is located at the propeller shaft plane.

The presence of a stratification interface against the solid surface of a hull has similar effects as those of a free surface in a juncture flow, where the boundary layer thickens due to the generation of streamwise vortices that take low velocity fluid from the boundary layer out from

the hull. In the case of the submarine with the density interface at shaft depth this causes a significant velocity deficit into the propeller, which deforms considerably the vortical structures in the propeller wake.

Future work will focus on study of far-field wake produced by maritime vehicles advancing in a stable stratified fluid.

\section{Acknowledgements}

This research was supported by the Office of Naval Research, grant N000141310351, with Drs. Ki-Han Kim and Patrick Purtell as program officers. Computations were performed in the SGI Ice-X Spirit at the Air Force Research Laboratory DoD supercomputer resource center and The University of Iowa computer cluster Helium. 


\section{References}

Al-Amiri, A.M., Khanafer K.M., Pop, I. (2007). Numerical simulation of combined thermal and mass transport in a square lid-driven cavity. Int. J. Thermal Sci. 46:662-671.

Balay, S., Brown, J., Buschelman, K, Eijkhout, V., Gropp, W., Kaushik, D., Knepley M., Curfman McInnes, L., Smith, B., Zhang, H. (2013). PETSc User Manual Revision 3.4.

Bonneton, P., Chomaz, J.M., Hopfinger, E.J. (1993). Internal waves produced by the turbulent wake of a sphere moving horizontally in a stratified fluid. J. Fluid Mech 254:23-40.

Boyer, D.L., Davies, P.A., Fernando, H.J.S., Zhang, X. (1989). Linearly stratified flow past a horizontal circular cylinder. Phil. Trans. R. Soc. London. A 328:501-528.

Brucker, K.A, Sarkar, S. (2010). A comparative study of self-propelled and towed wakes in a stratified fluid. J. Fluid Mech. 652:373-404.

Carrica, P.M., Wilson, R.V., Stern, F. (2007a). An unsteady single-phase level set method for viscous free surface flows. Int. J. Num. Meth. Fluids 53:229-56.

Carrica, P.M., Wilson, R.V., Noack, R., Stern, F. (2007b). Ship motions using single-phase level set with dynamic overset grids. Comput. Fluids 36:1415-33.

Carrica, P.M., Castro, A.M., Stern, F. (2010). Self-propulsion computations using a speed controller and a discretized propeller with dynamic overset grids. J. Mar. Sci. Technol. 15: 316-330.

Chang, Y., Zhao, F., Hong, F. W., Li, P., Yun, J. (2006). Numerical simulation of internal waves excited by a submarine in a two-layer stratified fluid. J. Hydrodynamics, Series B.18(3):330336.

Chase, N., Michael, T., Carrica, P.M. (2013). Overset simulations of a Submarine in Towed, Self-Propelled and Maneuvering Conditions. Int. Shipbuilding Progr. 60, 171-205.

Crapper, G. D. (1967). Ship waves in a stratified ocean. J. Fluid Mech. 29:667-672.

Crook, L.B. (1981). Powering Predictions for the R/V Athena (PG 94) Represented by Model 4950-1 with Design Propellers 4710 and 4711. David Taylor Naval Ship Research and Development Center report DTNSRDC/SPD-083305.

Ezz, H., Imran, J. (2014). Curvature-induced secondary flow in submarine channels. Environ. Fluid Mech. 14:343-370.

Fissel, D.B., Borg, K., Lemon, D.D., Brich, J.R. (2010). Marine Physical Environment, Enbridge Northern Gateway Project Technical Report, ASL Environmental Sciences Sidney, BC.

Hudimac, A.A. (1961). Ship waves in a stratified ocean. J. Fluid Mech. 11:229-243.

Ismail, F., Carrica, P. M., Xing, T., Stern, F. (2010). Evaluation of linear and nonlinear convection schemes on multidimensional non-orthogonal grids with applications to KVLCC2 tanker. Int. J. Num. Meth. Fluids, 64:850-886.

ITTC, (1978). Recommended Procedures and guidelines: 1978 ITTC Performance Prediction Method. International Towing Tank Conference Report 7.5-02-03-01.4.

Jackson, C.R., da Silva, J.C.B., Jeans, G., Alpers, W., Caruso, M.J. (2013). Nonlinear internal waves in synthetic aperture radar imagery. Oceanography 26:68-79. 
Jenkins, D.S. (1984). Resistance Characteristics of the High Speed Transom Stern Ship R/V Athena in the Bare Hull Condition, Represented by DTNSRDC Model 5365. David Taylor Research and Development Center report DTNSRDC-84/024.

Li, J., Castro, A.M., Carrica, P.M. (2015). A pressure-velocity coupling approach for high void fraction free surface bubbly flows in overset curvilinear grids. Int. J. Num. Meth. Fluids 79:343-369.

Lin, Q., Boyer, D.L., Fernando, J.S. (1992a). Turbulent wakes of linearly stratified flow past a sphere. Phys Fluids A 4:1687-1696.

Lin, Q., Lindberg, W.R., Boyer, D.L., Fernando, H.J.S. (1992b). Stratified flow past a sphere. J. Fluid Mech. 240:315-354.

Longo, J., Huang, H.P., Stern, F. (1998). Solid/free surface juncture boundary layer and wake. Exp. Fluids 25:283-297.

Martin, J.E., Michael, T., Carrica, P.M. (2015). Submarine maneuvers using direct overset simulation of appendages and propeller and coupled CFD/potential flow propeller solver. J. Ship Res. 59(1):31-48.

Menter, F.R. (1994). Two-Equation Eddy-Viscosity Turbulence Models for Engineering Applications. AIAA Journal 32(8):1598-1605.

Meunier, P., Spedding, G.R. (2004). A loss of memory in stratified momentum wakes. Phys. Fluids 16:298-305.

Meunier, P., Spedding, G.R. (2006). Stratified propelled wakes. J. Fluid Mech. 552:229-256.

Miloh, T., Tulin, M.P., Zilman G. (1993). Dead-water effects of a ship moving in stratified seas. J. Offshore Mech. Arctic Eng. 115:105-110.

Mofidi, A., Carrica, P.M. (2014). Simulations of zigzag maneuvers for a container ship with direct moving rudder and propeller. Comput. Fluids 96:191-203.

Noack, R. (2005). SUGGAR: a general capability for moving body overset grid assembly. In 17 th AIAA CFD Conf., Toronto, ON, Canada.

Noack, R., Boger, D., Kunz, R., Carrica P.M. (2009). Suggar++: An Improved General Overset Grid Assembly Capability. AIAA Paper 2009-3992, $19^{\text {th }}$ AIAA CFD Conf., San Antonio, TX.

Overpelt, B. (2015). Free Running Manoeuvring Model Tests on a Modern Generic SSK Class Submarine (BB2), PACIFIC 2015 Int. Maritime Conf., Sydney, NSW, Australia.

Serchi, F.G., Peakall, J., Ingham, D.B., Burns, A.D. (2012). A numerical study of the triggering mechanism of a lock-release density current. Euro. J. Mech. B/Fluids 33:25-39.

Shih, L., Koseff, J.R., Ivey, G.N., Ferziger, J.H. (2005). Parameterization of turbulent fluxes and scales using homogeneous sheared stably stratified turbulence simulations. J. Fluid Mech. 525:193-214.

Spedding, G.R., Browand, F.K., Fincham, A.M. (1996). Turbulence, similarity scaling and vortex geometry in the wake of a towed sphere in a stably stratified fluid. J. Fluid Mech. 314:53-103.

de Stadler, M.B., Sarkar, S., Brucker, K.A. (2010). Effect of the Prandtl number on a stratified turbulent wake. Phys. Fluids 22:095102 
Stern, F., Wilson, R.V., Coleman, H.V., Paterson, E.G. (2001). Comprehensive approach to verification and validation of CFD simulations - Part 1 and 2: methodology and procedures. ASME J. Fluids Eng. 124:793-802.

Tseng, Y.H, Ferziger, J.H. (2001). Mixing and available potential energy in stratified flows. Phys. Fluids 13(5):1281-1293.

Tulin, M.P., Yao, Y., Wang, P. (2000). The generation and propagation of ship internal waves in a generally stratified ocean at high densimetric Froude numbers, including nonlinear effects. J. Ship Res. 44(3):197-227.

Venayagamoorthy, S.K., Koseff J.R., Ferziger J.H., Shih L.H. (2003). Testing of RANS turbulence models for stratified flows based on DNS data," Center for Turbulence Research, Annual Research Briefs 127-38.

Watson, G., Chapman, R.D., Apel, J.R. (1992). Measurements of the Internal Wave Wake of a Ship in a Highly Stratified Sea Loch, J. Geophys. Res. 97(C6): 9689-9703.

Winters, K.B., Lombard, P.N., Riley, J.J., D'asaro, E.A. (1995). Available potential energy and mixing in density-stratified fluids. J. Fluid Mech. 289:115-128.

Yeoh, G.H., Tu J. (2010). Computational techniques for multi-phase flows. ButterworthHeinemann/ IChemE. 


\section{Table Captions:}

Table 1. Grid study for the density gradient and velocity along the vertical line at the center of the cavity in steady state.

Table 2. Time step study for density gradient and velocity along the vertical line at the center of the cavity at time $=10$.

Table 3. Main particulars of Athena R/V.

Table 4. Main particulars of Joubert BB2.

Table 5. Main particulars of the Joubert BB2 propeller, MARIN stock propeller 7371R.

Table 6. Comparison of Joubert BB2 performance in uniform and stratified flows at 10 knots. 
Table 1. Grid study for the density gradient and velocity along the vertical line at the center of the cavity in steady state.

\begin{tabular}{lcccccccc}
\hline Variable & $\begin{array}{c}\text { Coarse } \\
\text { Mesh }\end{array}$ & $\begin{array}{c}\text { Medium } \\
\text { Mesh }\end{array}$ & $\begin{array}{c}\text { Fine } \\
\text { Mesh }\end{array}$ & $\mathbf{R}_{\mathbf{G}}$ & $\mathbf{C}_{\mathbf{G}}$ & $\mathbf{P}_{\mathbf{G}}$ & $\mathbf{U}_{\mathbf{G C}}$ & $\begin{array}{c}\text { Convergence } \\
\text { type }\end{array}$ \\
\hline$\left(\frac{d \rho}{d z}\right)_{\text {Bottom wall }}$ & 7.632039 & 7.625449 & 7.624179 & 0.19 & 1.34 & 2.37 & $1.2 \mathrm{E}-4$ & Monotonic \\
$\left(\frac{d \rho}{d z}\right)_{\text {Top wall }}$ & 3.913535 & 3.836199 & 3.814099 & 0.29 & 0.84 & 1.81 & $1.48 \mathrm{E}-3$ & Monotonic \\
$U_{z, \text { max }}$ & 0.065180 & 0.065665 & 0.065813 & 0.31 & 0.76 & 1.71 & $1.57 \mathrm{E}-5$ & Monotonic \\
\hline
\end{tabular}


Table 2. Time step study for density gradient and velocity along the vertical line at the center of the cavity at time $=10$.

\begin{tabular}{lcccccccc}
\hline Variable & $\Delta t=0.02$ & $\Delta t=0.01$ & $\Delta t=0.005$ & $\mathbf{R}_{\mathbf{T}}$ & $\mathbf{C}_{\mathbf{T}}$ & $\mathbf{P}_{\mathbf{T}}$ & $\mathbf{U}_{\mathbf{T C}}$ & $\begin{array}{c}\text { Convergence } \\
\text { type }\end{array}$ \\
\hline$\left(\frac{d \rho}{d z}\right)_{\text {Bottom wall }}$ & 3.136232 & 3.136546 & 3.136651 & 0.333 & 0.666 & 1.59 & $1.75 \mathrm{E}-5$ & Monotonic \\
$\left(\frac{d \rho}{d z}\right)_{\text {Top wall }}$ & 7.862150 & 7.861966 & 7.861895 & 0.382 & 0.538 & 1.39 & $2.01 \mathrm{E}-5$ & $\begin{array}{c}\text { Monotonic } \\
U_{z, \text { max }}\end{array}$ \\
\hline & 0.103577 & 0.103571 & 0.103570 & 0.244 & 1.034 & 2.03 & $4.77 \mathrm{E}-8$ & Monotonic \\
\hline$\left(\frac{d \rho}{d z}\right)_{\text {Bottom wall }}$ & 3.134871 & 3.136232 & 3.136546 & 0.231 & 1.111 & 2.11 & $1.22 \mathrm{E}-5$ & Monotonic \\
$\left(\frac{d \rho}{d z}\right)_{\text {Top wall }}$ & 7.862702 & 7.862150 & 7.861966 & 0.34 & 0.67 & 1.6 & $3.07 \mathrm{E}-5$ & Monotonic \\
$U_{z, \text { max }}$ & 0.103597 & 0.103577 & 0.103571 & 0.303 & 0.765 & 1.71 & $6.05 \mathrm{E}-7$ & Monotonic \\
\hline
\end{tabular}


Table 3. Main particulars of Athena R/V.

\begin{tabular}{lc}
\hline Length overall submerged & $47.2 \mathrm{~m}$ \\
Beam & $7.16 \mathrm{~m}$ \\
Maximum draft & $3.2 \mathrm{~m}$ \\
Top speed & $35 \mathrm{~m}$ \\
Maximum shaft power & $9700 \mathrm{~kW}$ \\
Design displacement & 245 tons \\
\hline
\end{tabular}


Table 4. Main particulars of Joubert BB2.

Length overall submerged

$70.2 m$

Beam

$9.6 \mathrm{~m}$

Depth (to deck)

$10.6 \mathrm{~m}$

Depth (to top of sail)

$16.2 \mathrm{~m}$ 
Table 5. Main particulars of the Joubert BB2 propeller, MARIN stock propeller 7371R.

\begin{tabular}{lc}
\hline Number of blades & 6 \\
Propeller diameter & $5.0 \mathrm{~m}$ \\
Hub diameter & $1.114 \mathrm{~m}$ \\
Hub/diameter ratio & 0.223 \\
Pitch/diameter at $0.7 \mathrm{R}$ & 0.966 \\
Pitch at $0.7 \mathrm{R}$ & $4.83 \mathrm{~m}$ \\
Expanded blade area ratio & 0.7 \\
\hline
\end{tabular}


Table 6. Comparison of Joubert BB2 performance in uniform and stratified flows at 10 knots.

\begin{tabular}{lccc}
\hline Parameter & \multicolumn{2}{c}{ Stratified } & Constant \\
\cline { 2 - 3 } & $z_{0}=-0.25 L_{0}$ & $z_{0}=-0.33 L_{0}$ & density \\
\hline Effective power $P_{E}(\mathrm{~kW})$ & 443.5 & 469.0 & 434.8 \\
Propeller rotational speed (RPS) & 0.978 & 0.99 & 0.966 \\
Propeller torque $Q(\mathrm{kN}-\mathrm{m})$ & 80.3 & 83.9 & 78.6 \\
Propeller thrust $T(\mathrm{kN})$ & 86.2 & 91.2 & 84.5 \\
Shaft power $P_{p}(\mathrm{~kW})$ & 493.6 & 521.3 & 477.6 \\
Advance velocity (m/s), thrust id. method & 3.52 & 3.52 & 3.48 \\
Propeller efficiency (\%), thrust id. method & 61.6 & 61.5 & 61.5 \\
Extra power due to stratification (kW) & 8.7 & 34.2 & --- \\
Estimated potential energy flux (Eq. 10, & 7.3 & 33.5 & -- \\
$\mathrm{kW}$ ) & & & \\
\hline
\end{tabular}




\section{Figure Captions:}

Figure 1. Boundary conditions for square cavity with density gradient.

Figure 2. U velocity profile at the vertical center line (left) and contours (right) for the lid-driven cavity.

Figure 3. Density profile at the vertical center line (left) and contours (right) for the lid-driven cavity.

Figure 4. Overset grid system for the towed sphere problem.

Figure 5. Experimental visualization (top, reproduced from Bonneton et al., 1993) and CFD (bottom) results of the vertical displacement $\zeta$ related to the lee wave in a horizontal plane at $|\mathrm{z}|=3 \mathrm{R}$ below the center of the sphere for $F i=10 / \pi$ and $R e=805$.

Figure 6. Visualization of the temporal evolution of the internal waves in a horizontal plane $|z|=3 R$ below the center of the sphere. The scale is $46.5 \mathrm{R} \times 32 \mathrm{R}$. EFD panels reproduced from Bonneton et al. (1993).

Figure 7. Wavelength of the random wave as a function of $N t$, and normalized with the sphere diameter. Different internal Froude numbers are shown. Experimental data of Bonneton et al. (1993) has $\operatorname{Re}(1)=2614$ and current CFD data has $\operatorname{Re}(1)=253$.

Figure 8. Internal wave amplitude as a function of the internal Froude number, measured at $|\mathrm{z}|=3 \mathrm{R}$ above the center of the sphere and 80R downstream. $\operatorname{Re}(1)=2614$ for the experimental data of Bonneton et al. (1993) and $\operatorname{Re}(1)=253$ for current CFD results.

Figure 9. The geometry and grid topology for Athena R/V.

Figure 10. Density profile used for ship and submarine simulations. Left: measurements from Kitimat Bay station (full circles, Fissel et al. 2010); an analytical profile was adjusted to the data, with emphasis on capturing the appropriate density gradient in the region of operation of the vessel. A stronger than measured pycnocline was considered. Uniform density far from the vessel was adopted to improve numerical performance at the boundary. Neither of these two changes is expected to affect the solution of the near wake. Right: hyperbolic tangent profiles used for the Joubert BB2 simulations. The dashed line has maximum gradient at $z_{0}=-0.25 L_{0}$, approximately the propeller shaft centerline; the solid line has maximum gradient at $z_{0}=$ $-0.33 L_{0}$, slightly below the tail fins.

Figure 11. Instantaneous (top) and mean (bottom) density distributions at the center plane $\left(y / L_{0}=0\right)$ and at the starboard propeller plane $\left(y / L_{0}=0.0385\right)$. Turbulence model used indicated in each panel.

Figure 12. Axial cross sections for the self-propelled Athena $\mathrm{R} / \mathrm{V}$ at different axial cross sections downstream of the stern of the ship (only starboard side shown). Mean density (top) and mean longitudinal velocity (bottom) are shown. Passive scalar transport with DDES (left), stratified conditions with DDES (center) and stratified conditions using RANS (right). 
Figure 13. Axial cross sections for the self-propelled Athena $\mathrm{R} / \mathrm{V}$ at different axial cross sections downstream of the stern of the ship (only starboard side shown). Mean modeled (top) and resolved (bottom) turbulent kinetic energy are shown. Passive scalar transport with DDES (left), stratified conditions with DDES (center) and stratified conditions using RANS (right).

Figure 14. Joubert BB2 hull form and overset grid topology.

Figure 15. Joubert BB2 Self-propelled. Top: pycnocline at the propeller shaft centerline; bottom: pycnocline below the submarine. Free surface elevation, isosurfaces of $\mathrm{Q}=500$, colored with longitudinal velocity and density at different axial cross sections are shown. Inset: hull colored with density and streamlines showing tightening of the density interface.

Figure 16. Self-propelled Joubert BB2. Isosurfaces of $\rho=\rho_{0}+\Delta \rho / 2$ colored with elevation difference with respect to the background for the pycnocline at the propeller shaft (top), for the pycnocline below the submarine (center) and for a passive scalar (bottom). Isosurfaces of $\mathrm{Q}=$ 500 near the propeller are shown in the insets.

Figure 17: Mean velocity field 0.12 ship lengths downstream from the propeller for the selfpropelled Joubert BB2. Left column: stratified case with the pycnocline at the propeller shaft; right column: non-stratified case. The top panels show mean longitudinal velocity; center panels show resolved turbulent kinetic energy; bottom panels show modeled turbulent kinetic energy. The position of the $\rho=\rho_{0}+\Delta \rho / 2$ contour is shown as a solid black line.

Figure 18: Density field 0.12 ship lengths downstream from the propeller for the self-propelled Joubert BB2, for stratified case with the pycnocline at the propeller shaft. Left: mean density; right: mean vertical density gradient, expressed as buoyancy frequency.

Figure 19: Top left: mean Q in the boundary layer of the Joubert BB2 submarine for the stratified case with the interface at the propeller shaft; top right: same for non-stratified case. Bottom left: mean longitudinal velocity on axial cross sections showing the boundary layer; bottom right: boundary layer and transverse streamlines in the mid-ships plane; three isosurfaces of mean density are also shown.

Figure 20. Potential power flux $I_{x}$ as defined by Eq. (10), for the self-propelled Joubert BB2. Top: pycnocline at $z_{0}=-0.33 L_{0}$; bottom: pycnocline at $z_{0}=-0.25 L_{0}$. 


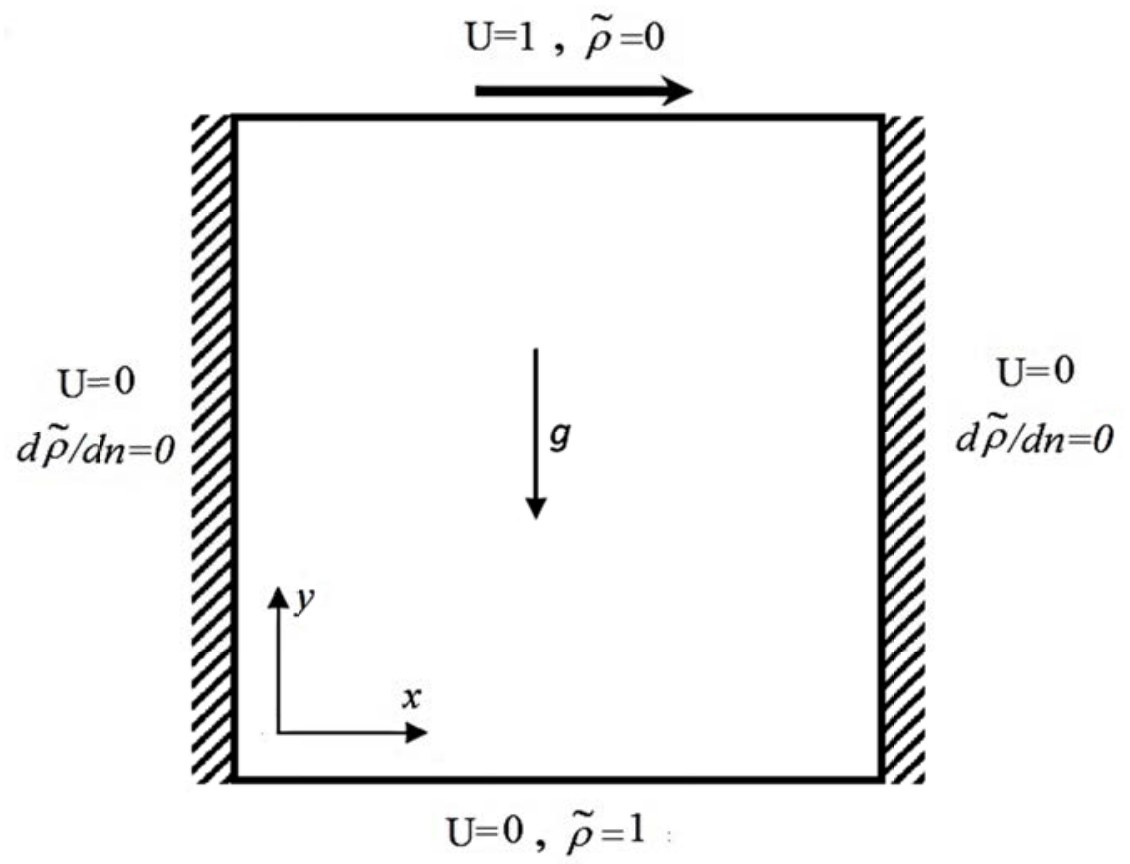

Figure 1. Boundary conditions for square cavity with density gradient. 


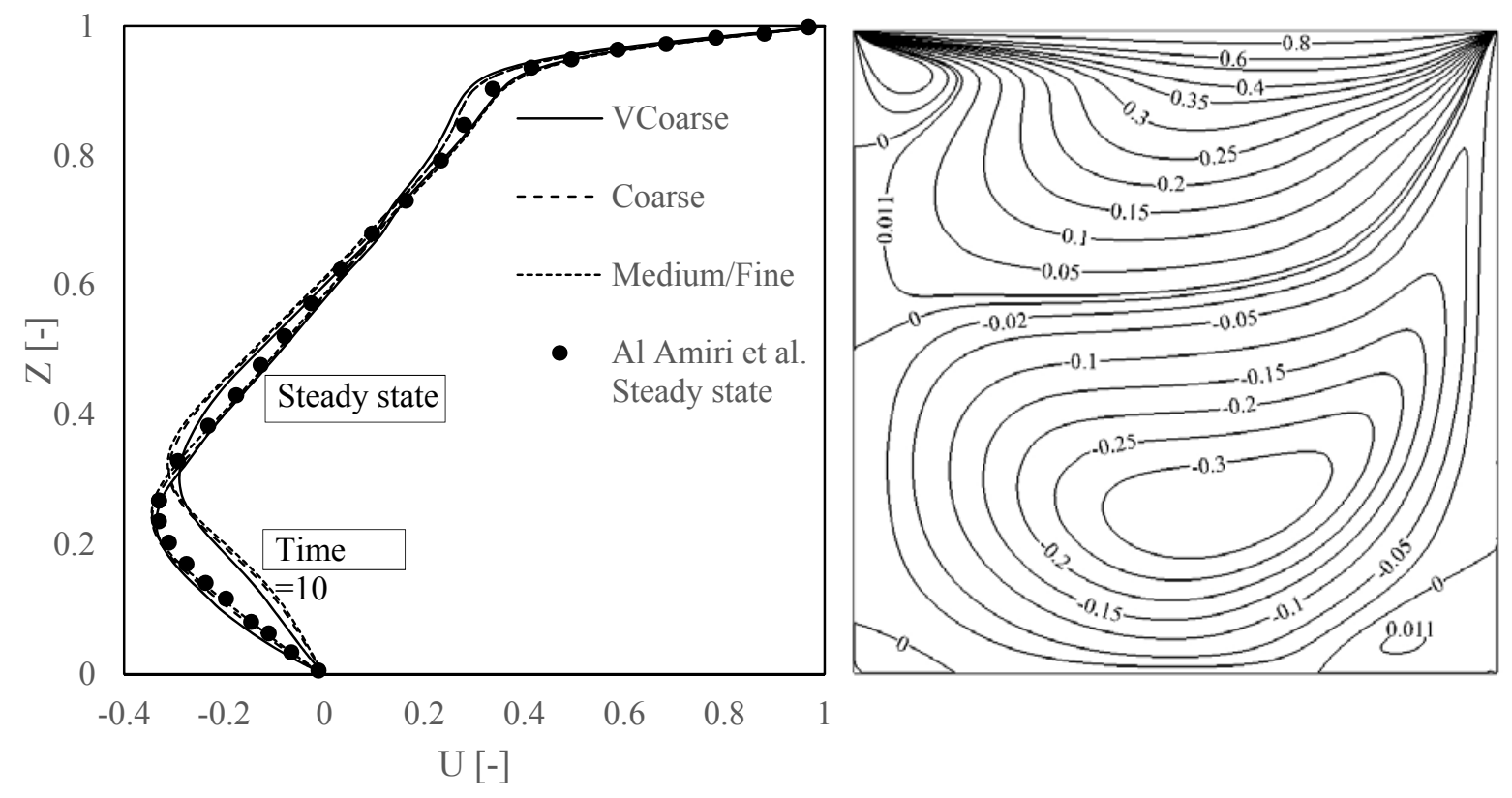

Figure 2. U velocity profile at the vertical center line (left) and contours (right) for the liddriven cavity. 

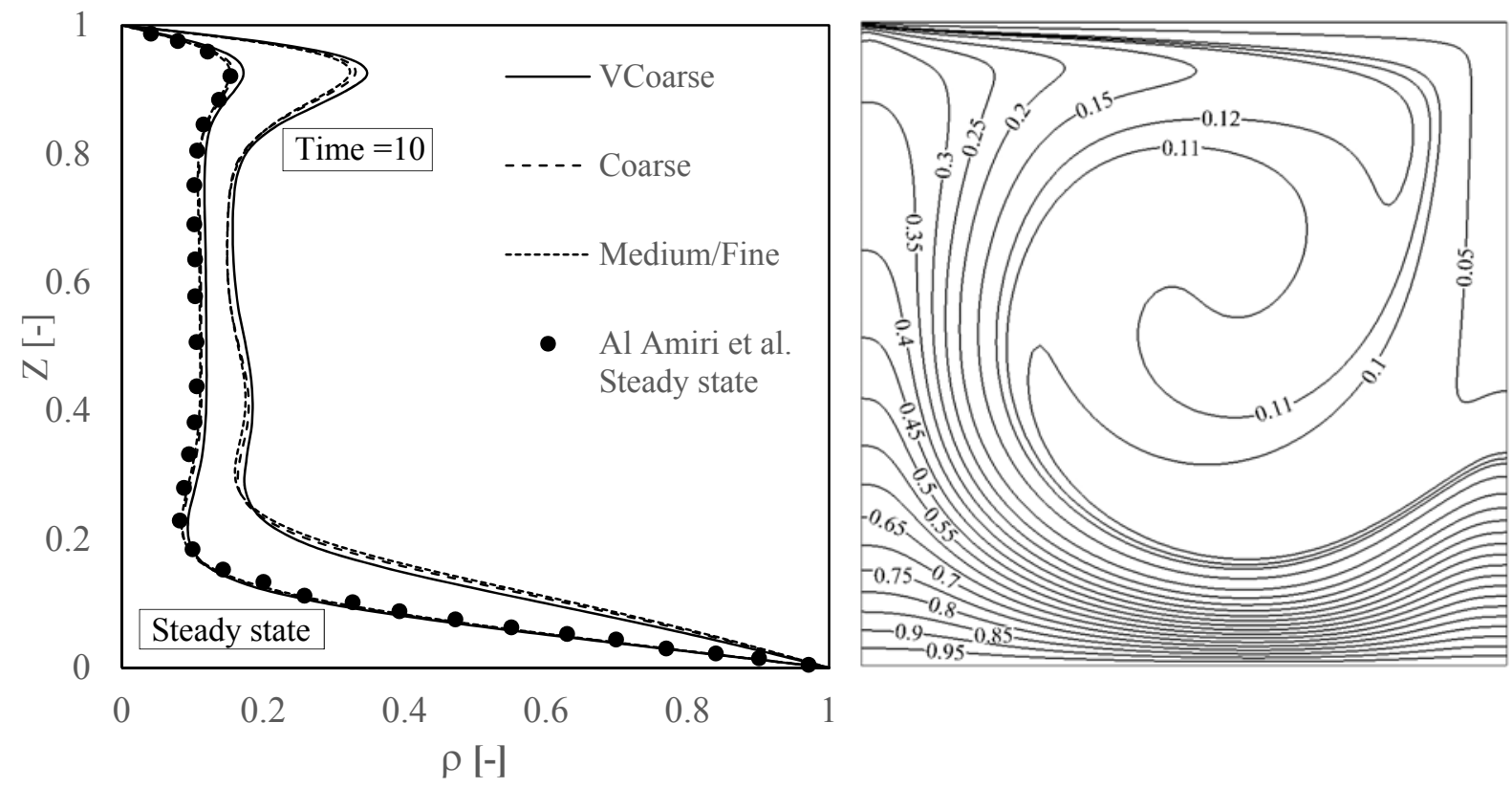

Figure 3. Density profile at the vertical center line (left) and contours (right) for the lid-driven cavity. 


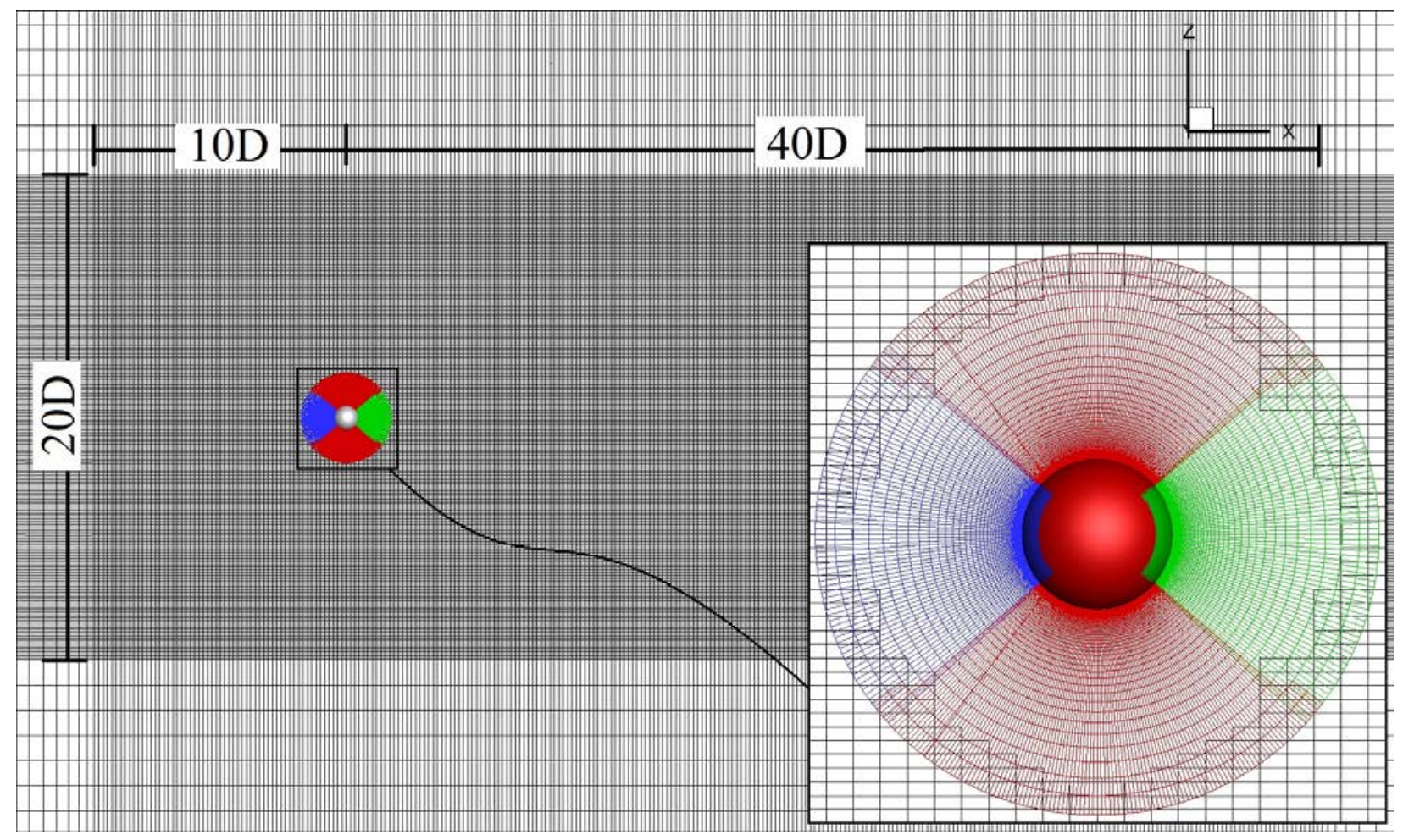

Figure 4. Overset grid system for the towed sphere problem. 


$$
N t=0.0
$$

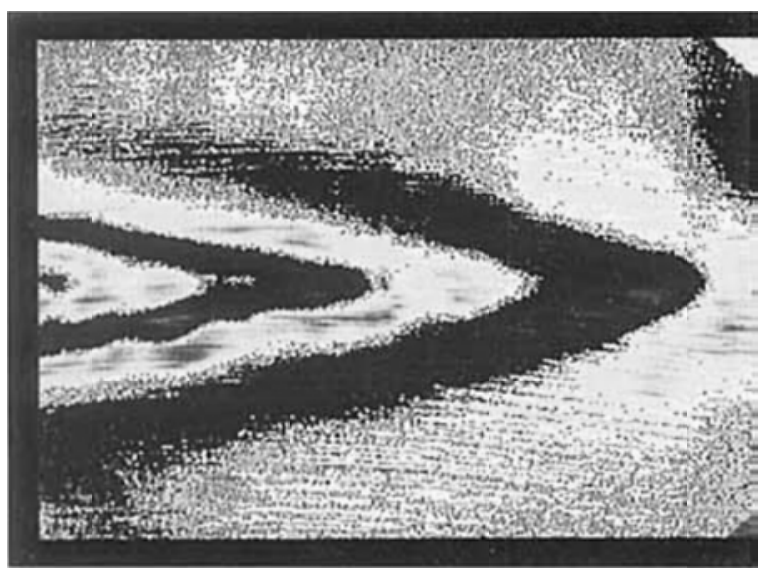

(a)

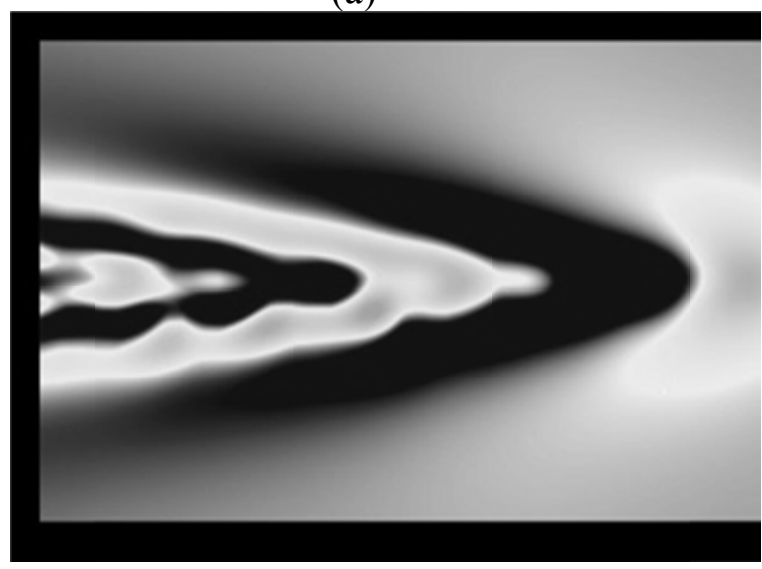

(c)

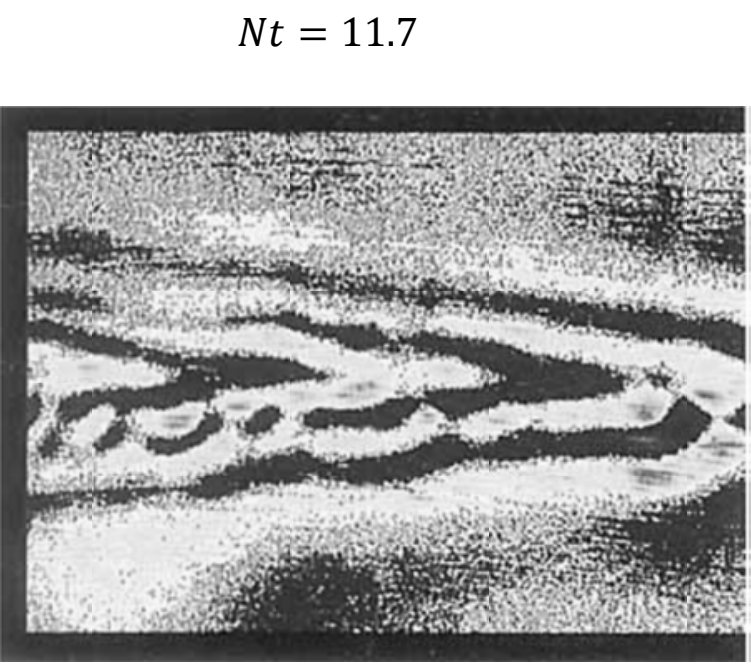

(b)

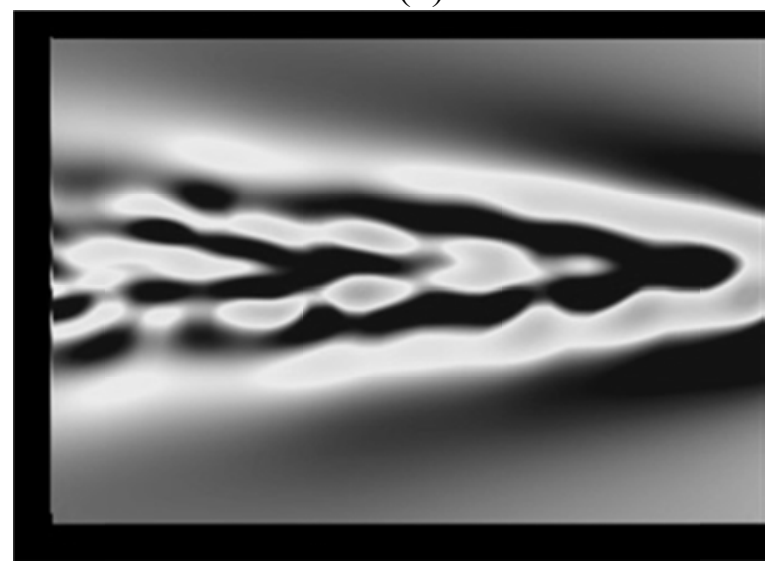

(d)

Figure 5. Experimental visualization (top, reproduced from Bonneton et al., 1993) and CFD (bottom) results of the vertical displacement $\zeta$ related to the lee wave in a horizontal plane at $|z|=3 R$ below the center of the sphere for $F i=10 / \pi$ and $R e=805$. 


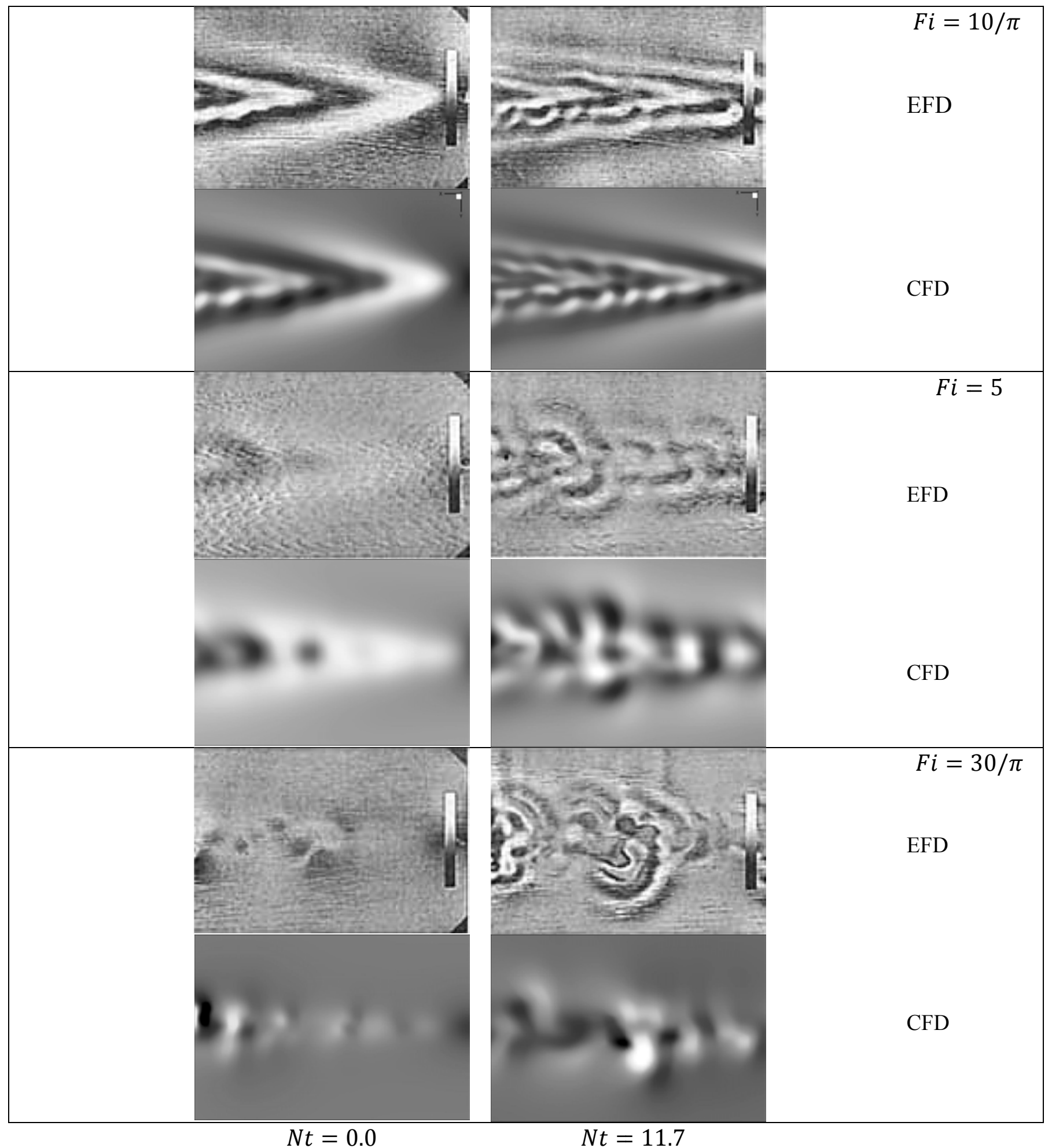

Figure 6. Visualization of the temporal evolution of the internal waves in a horizontal plane $|z|=3 R$ below the center of the sphere. The scale is $46.5 R \times 32 R$. EFD panels reproduced from Bonneton et al. (1993). 


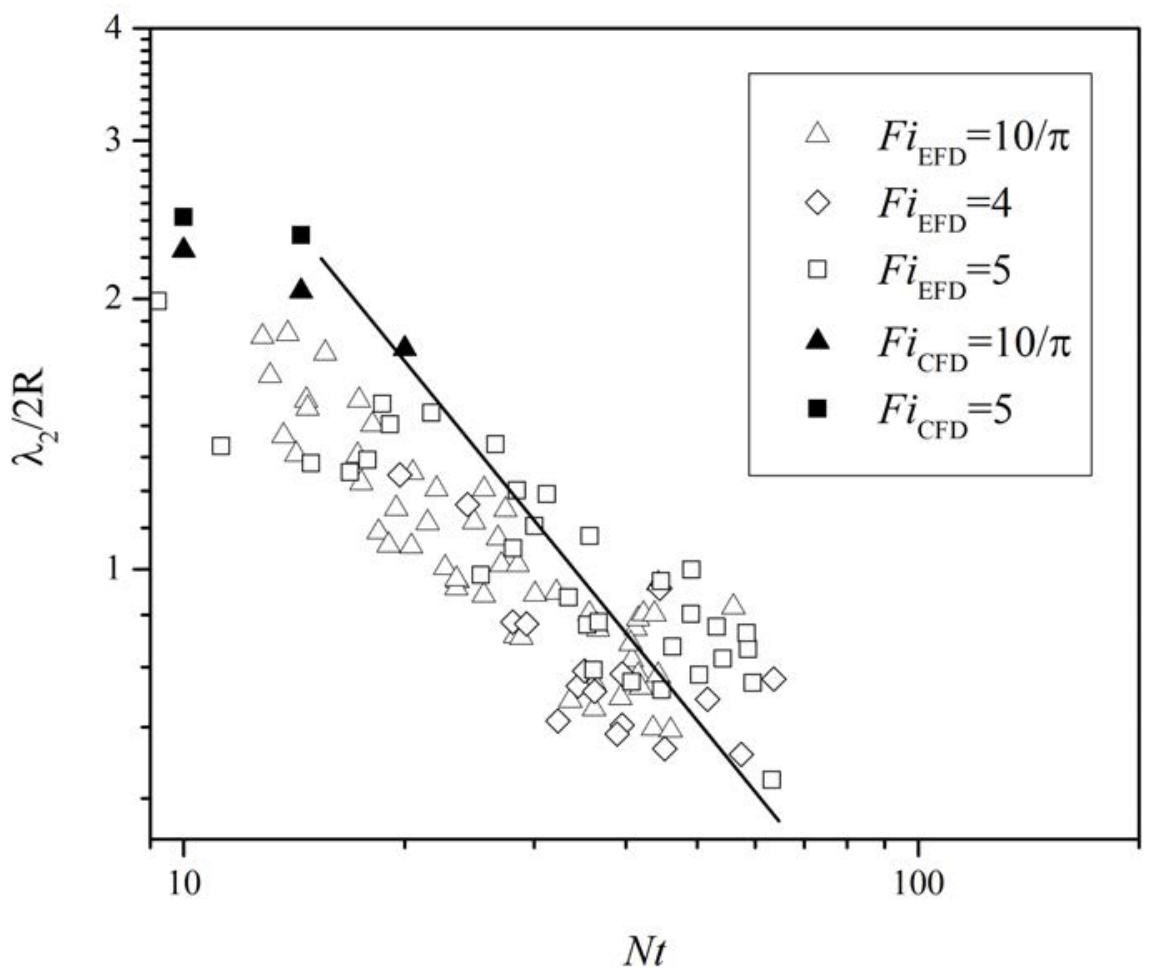

Figure 7. Wavelength of the random wave as a function of $N t$, and normalized with the sphere diameter. Different internal Froude numbers are shown. Experimental data of Bonneton et al. (1993) has $\operatorname{Re}(1)=2614$ and current CFD data has $\operatorname{Re}(1)=253$. 


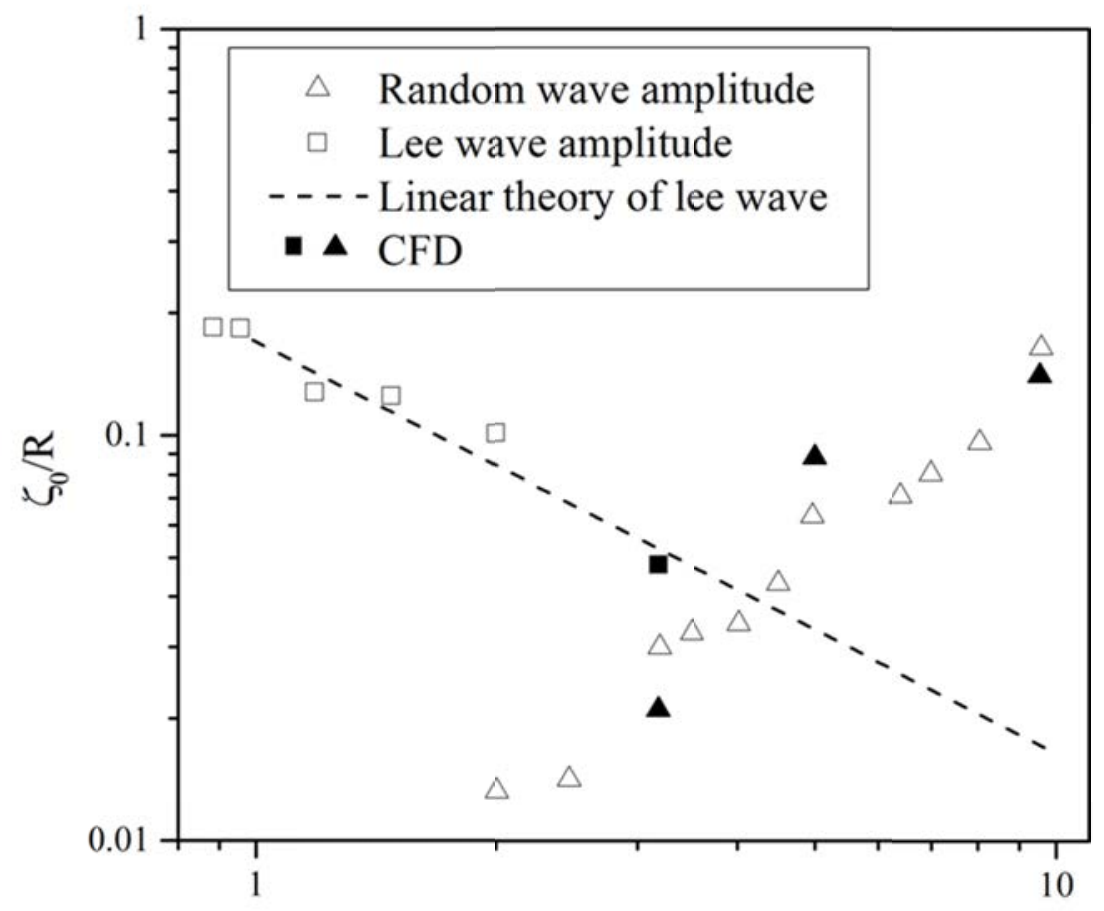

Fi

Figure 8. Internal wave amplitude as a function of the internal Froude number, measured at $|z|=3 R$ above the center of the sphere and 80R downstream. $\operatorname{Re}(1)=2614$ for the experimental data of Bonneton et al. (1993) and $\operatorname{Re}(1)=253$ for current CFD results. 

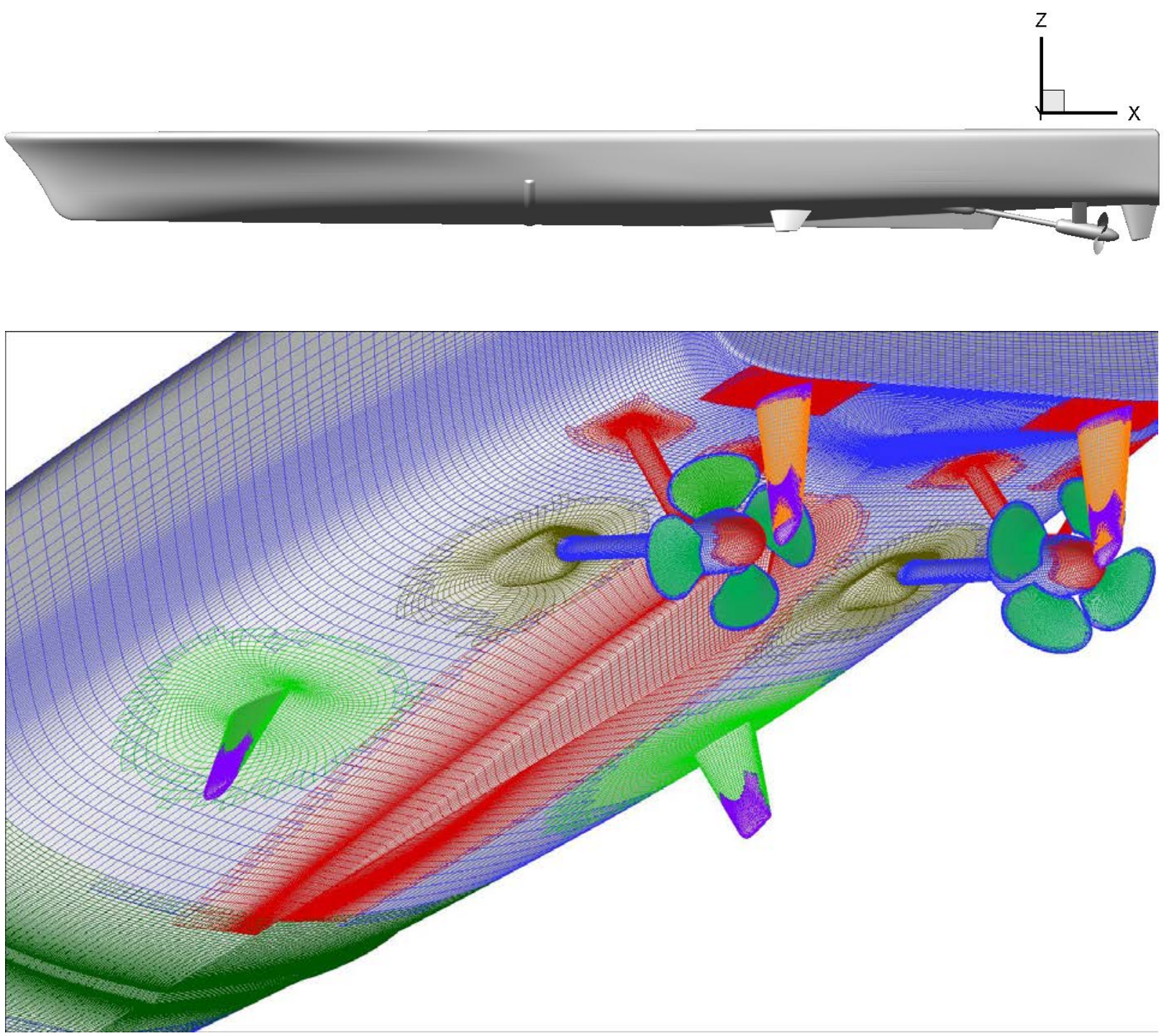

Figure 9. The geometry and grid topology for Athena R/V. 

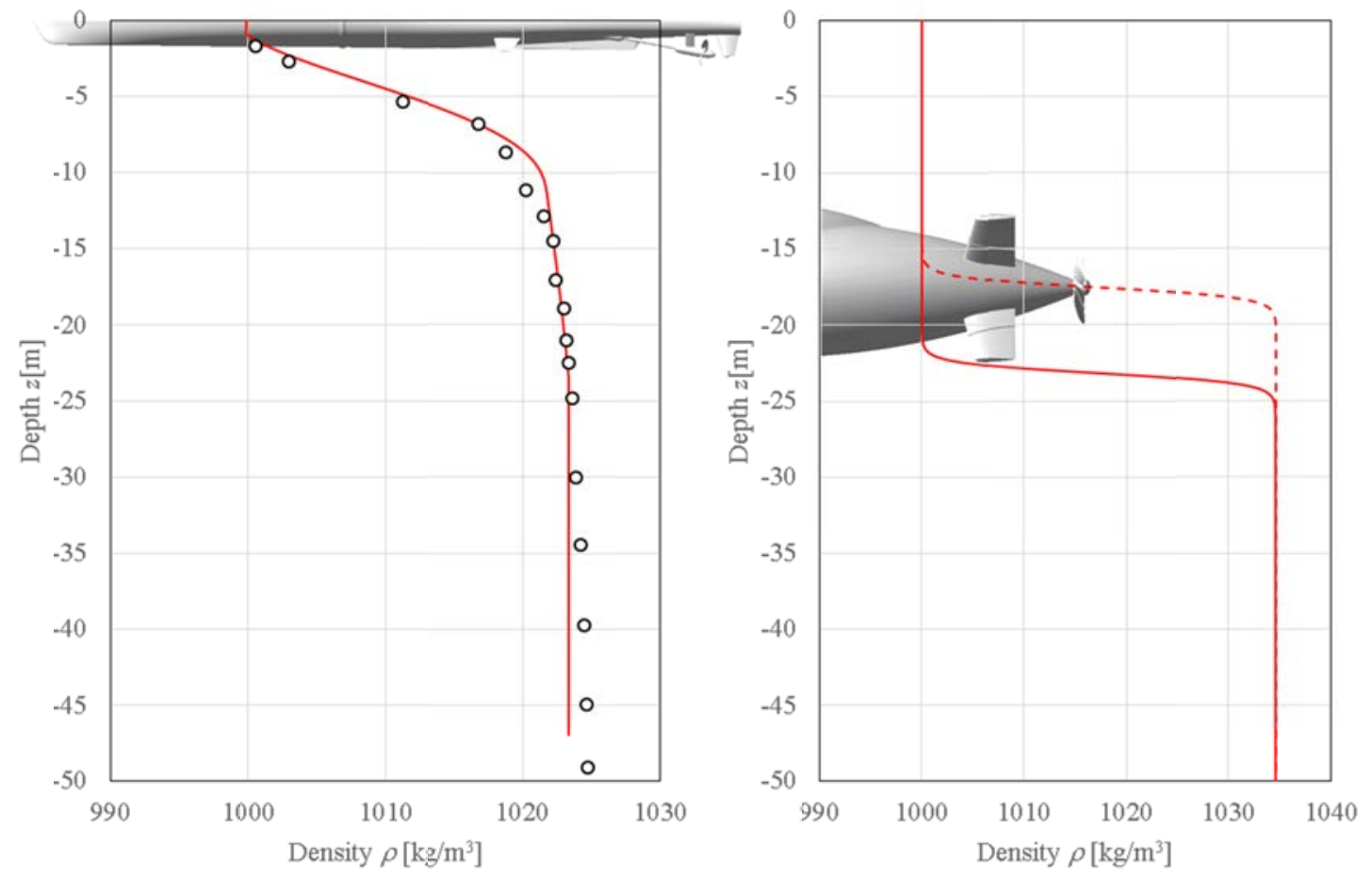

Figure 10. Density profile used for ship and submarine simulations. Left: measurements from Kitimat Bay station (full circles, Fissel et al. 2010); an analytical profile was adjusted to the data, with emphasis on capturing the appropriate density gradient in the region of operation of the vessel. A stronger than measured pycnocline was considered. Uniform density far from the vessel was adopted to improve numerical performance at the boundary. Neither of these two changes is expected to affect the solution of the near wake. Right: hyperbolic tangent profiles used for the Joubert BB2 simulations. The dashed line has maximum gradient at $z_{0}=-0.25 L_{0}$, approximately the propeller shaft centerline; the solid line has maximum gradient at $z_{0}=$ $-0.33 L_{0}$, slightly below the tail fins. 

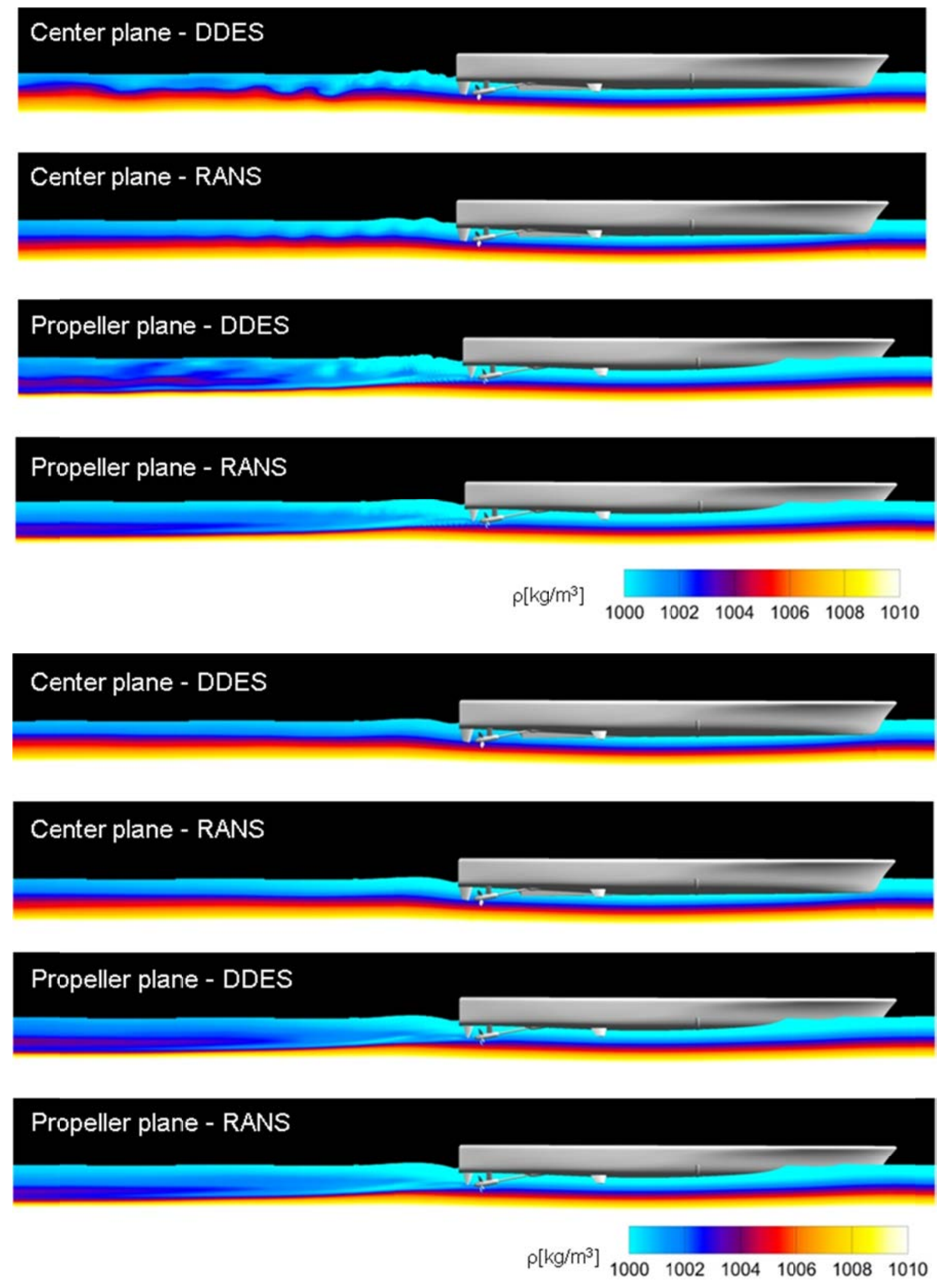

Figure 11. Instantaneous (top) and mean (bottom) density distributions at the center plane $\left(y / L_{0}=0\right)$ and at the starboard propeller plane $\left(y / L_{0}=0.0385\right)$. Turbulence model used indicated in each panel. 

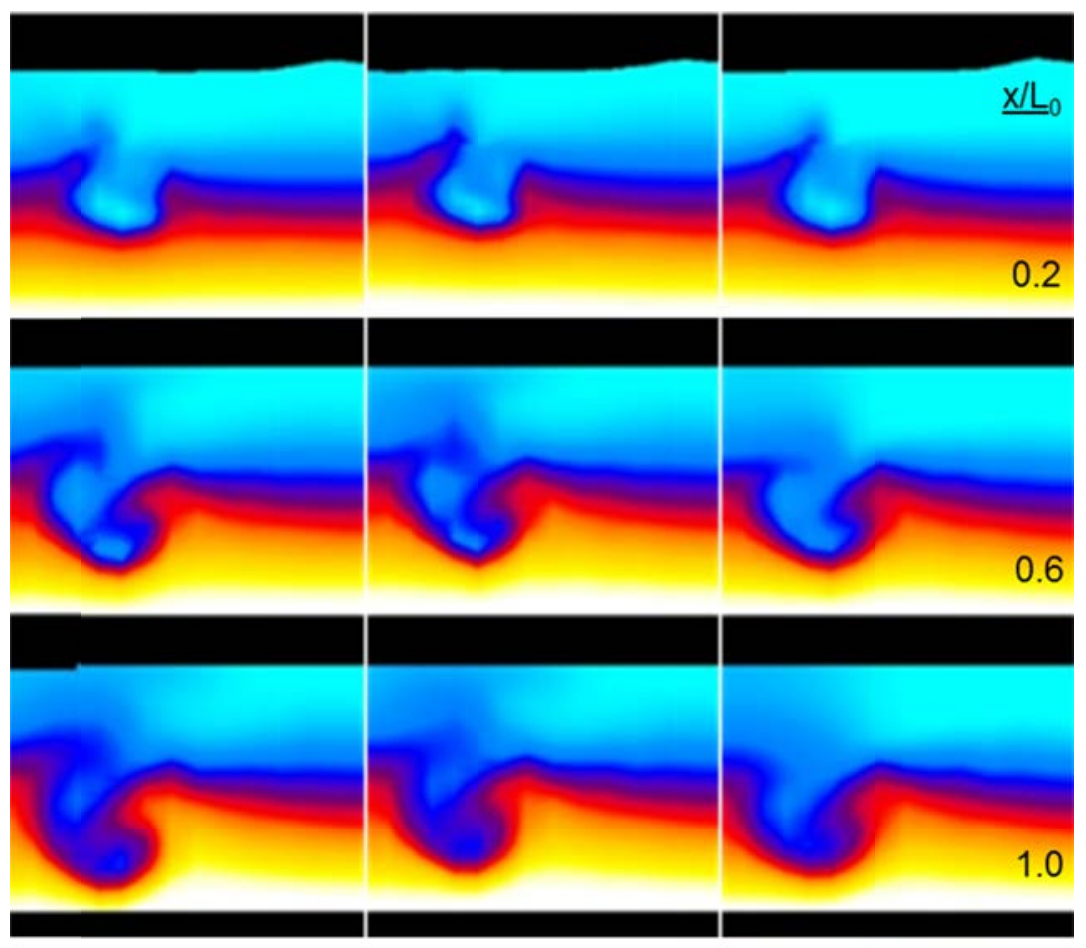

$\rho\left[\mathrm{kg} / \mathrm{m}^{3}\right]$

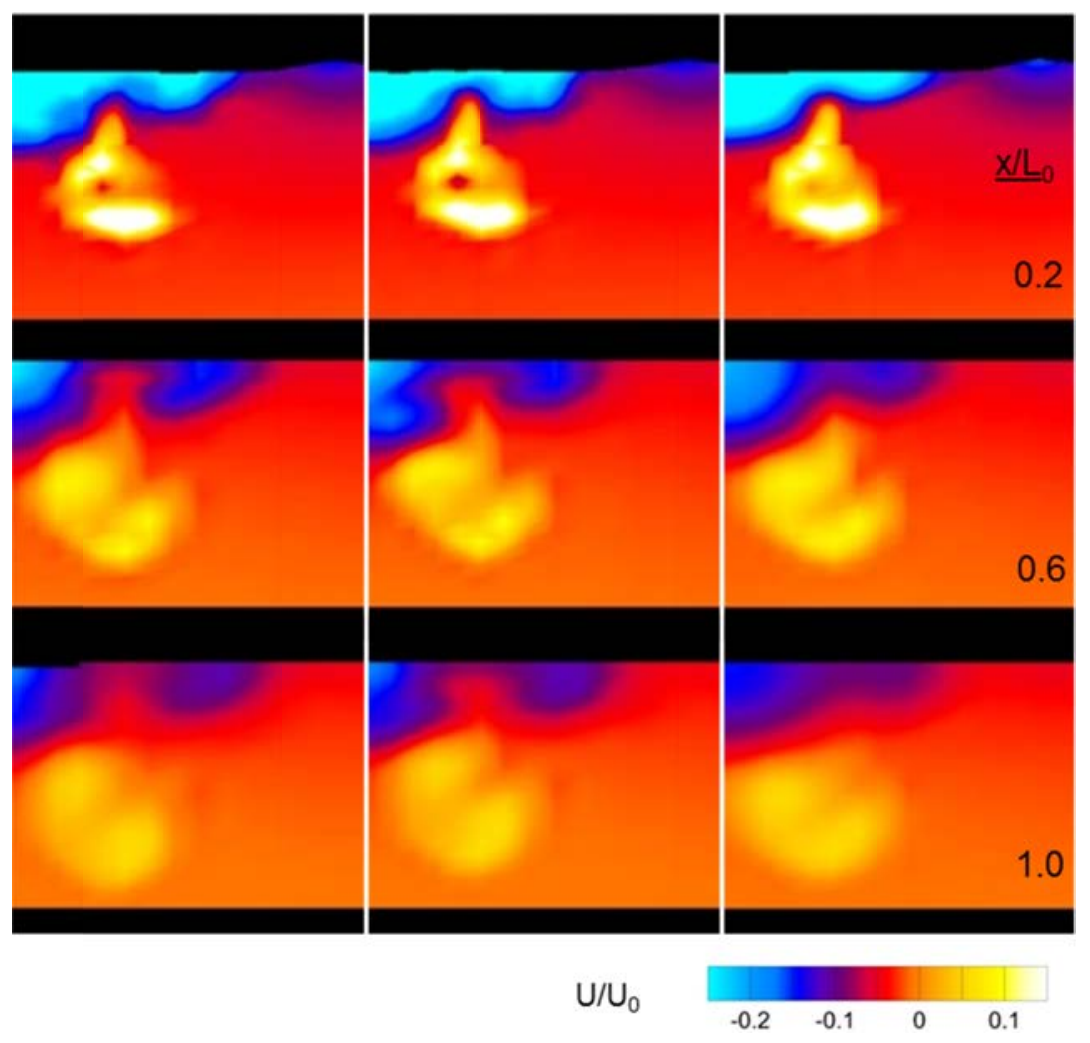

Figure 12. Axial cross sections for the self-propelled Athena $\mathrm{R} / \mathrm{V}$ at different axial cross sections downstream of the stern of the ship (only starboard side shown). Mean density (top) and mean longitudinal velocity (bottom) are shown. Passive scalar transport with DDES (left), stratified conditions with DDES (center) and stratified conditions using RANS (right). 

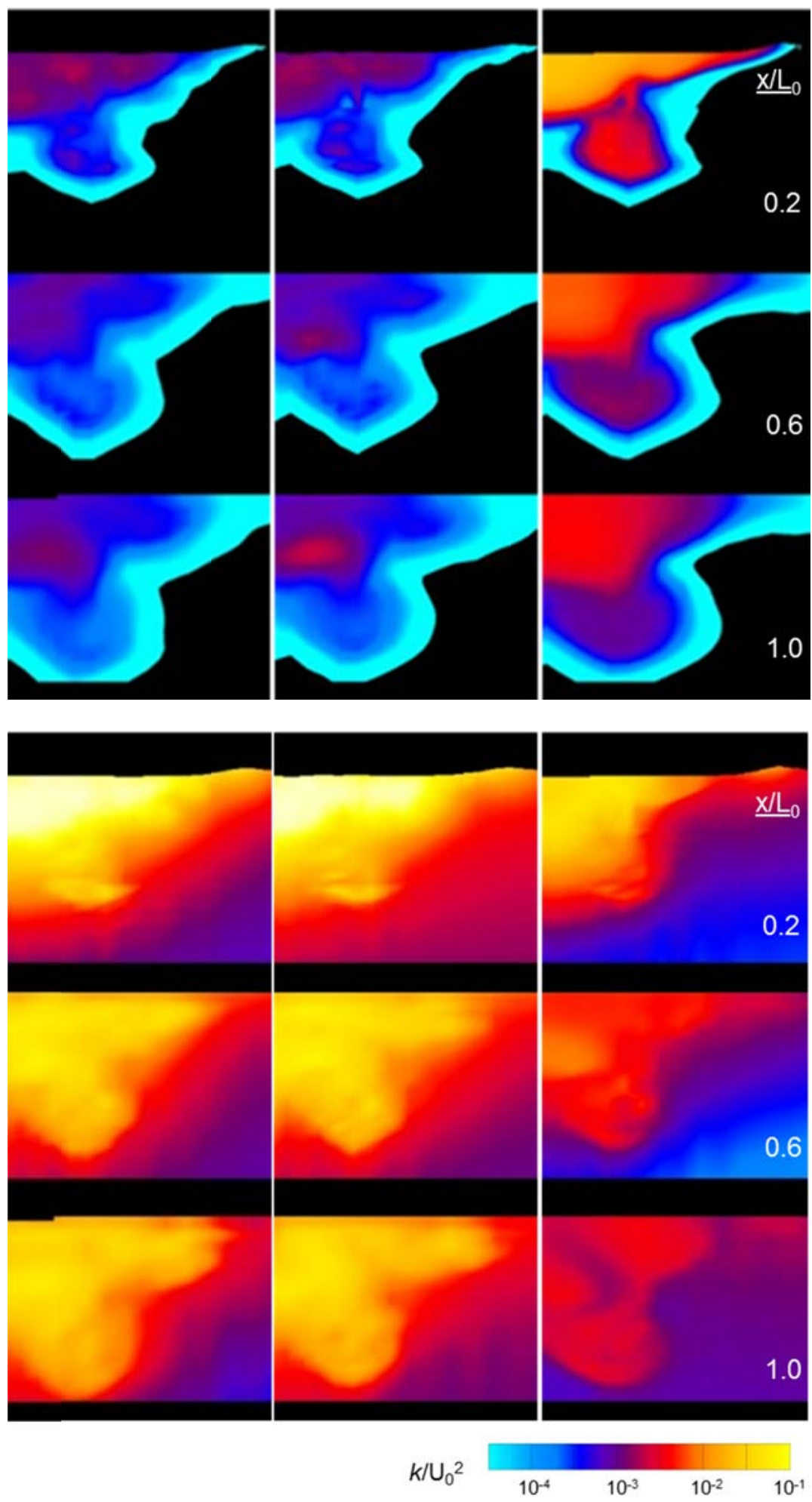

Figure 13. Axial cross sections for the self-propelled Athena $R / V$ at different axial cross sections downstream of the stern of the ship (only starboard side shown). Mean modeled (top) and resolved (bottom) turbulent kinetic energy are shown. Passive scalar transport with DDES (left), stratified conditions with DDES (center) and stratified conditions using RANS (right). 


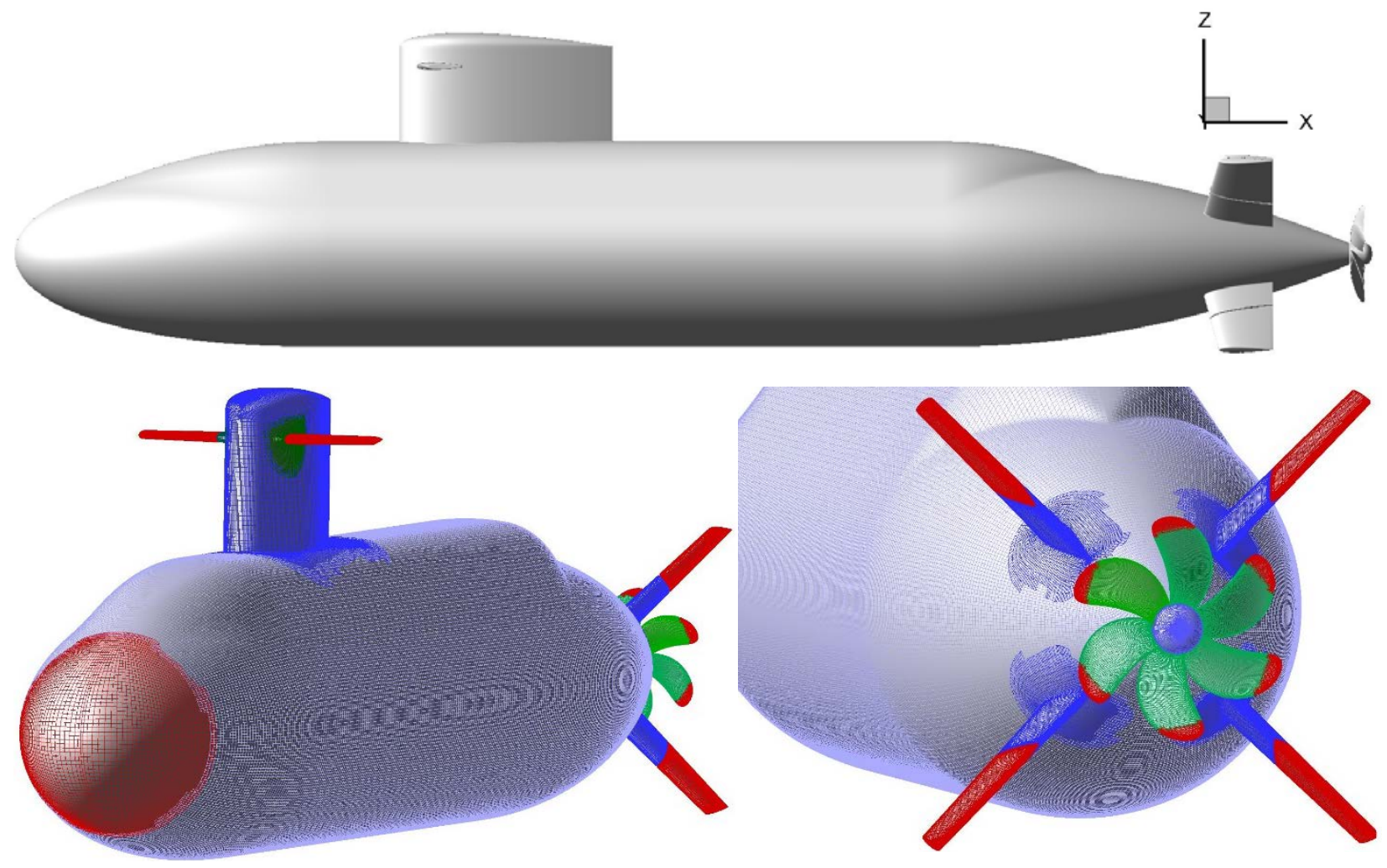

Figure 14. Joubert BB2 hull form and overset grid topology. 

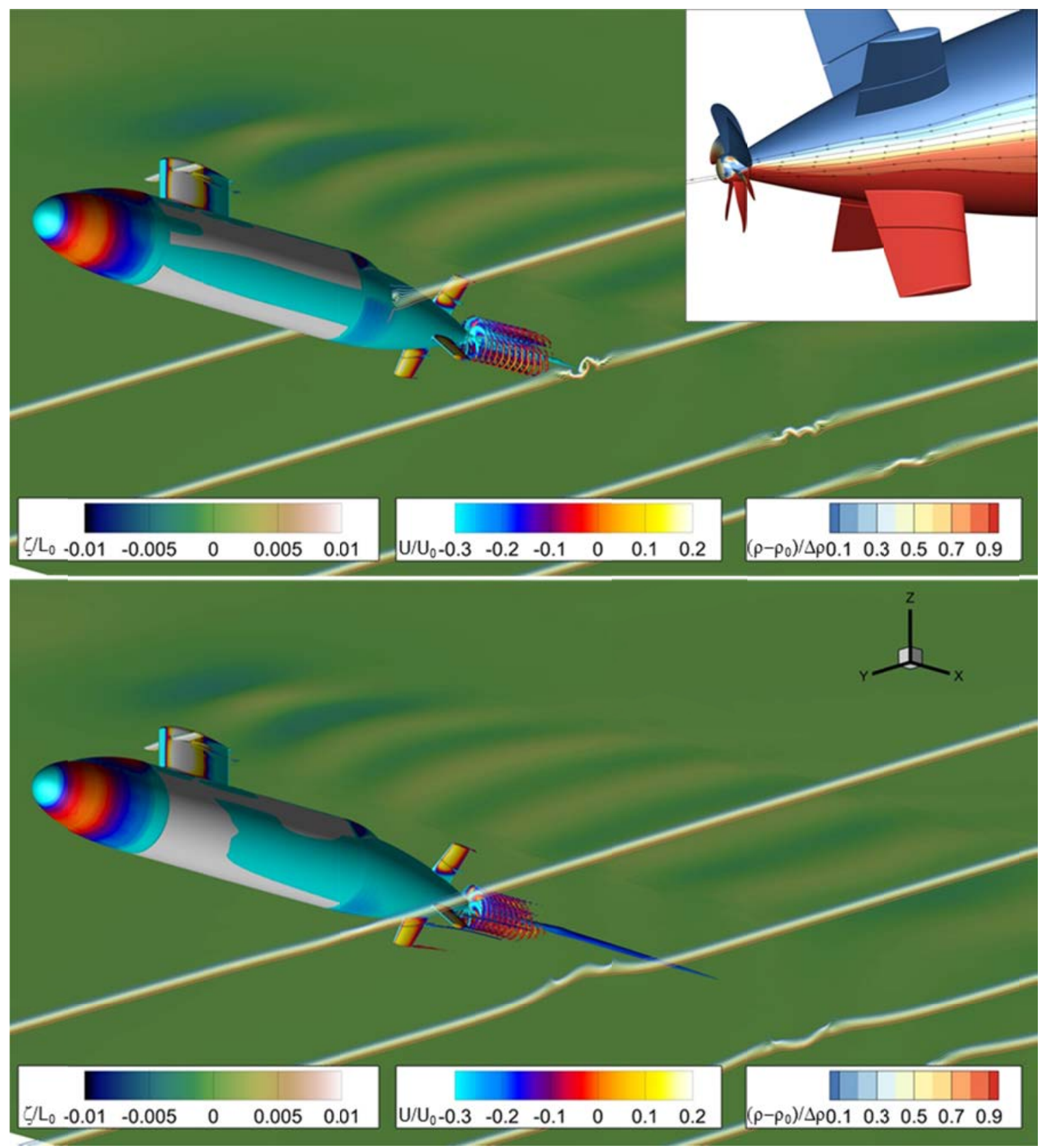

Figure 15. Joubert BB2 Self-propelled. Top: pycnocline at the propeller shaft centerline; bottom: pycnocline below the submarine. Free surface elevation, isosurfaces of $Q=500$, colored with longitudinal velocity and density at different axial cross sections are shown. Inset: hull colored with density and streamlines showing tightening of the density interface. 


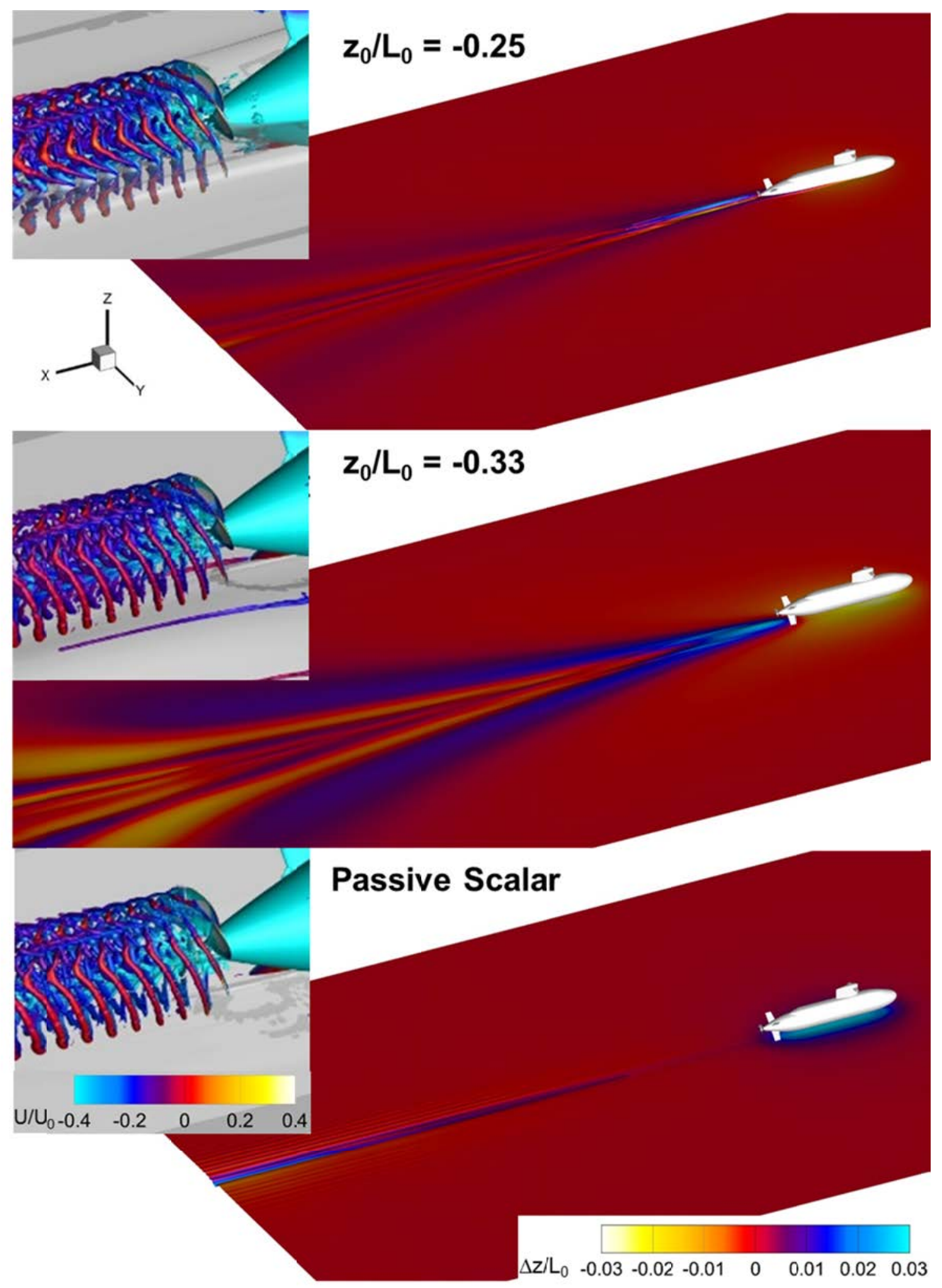

Figure 16. Self-propelled Joubert BB2. Isosurfaces of $\rho=\rho_{0}+\Delta \rho / 2$ colored with elevation difference with respect to the background for the pycnocline at the propeller shaft (top), for the pycnocline below the submarine (center) and for a passive scalar (bottom). Isosurfaces of $\mathrm{Q}=$ 500 near the propeller are shown in the insets. 

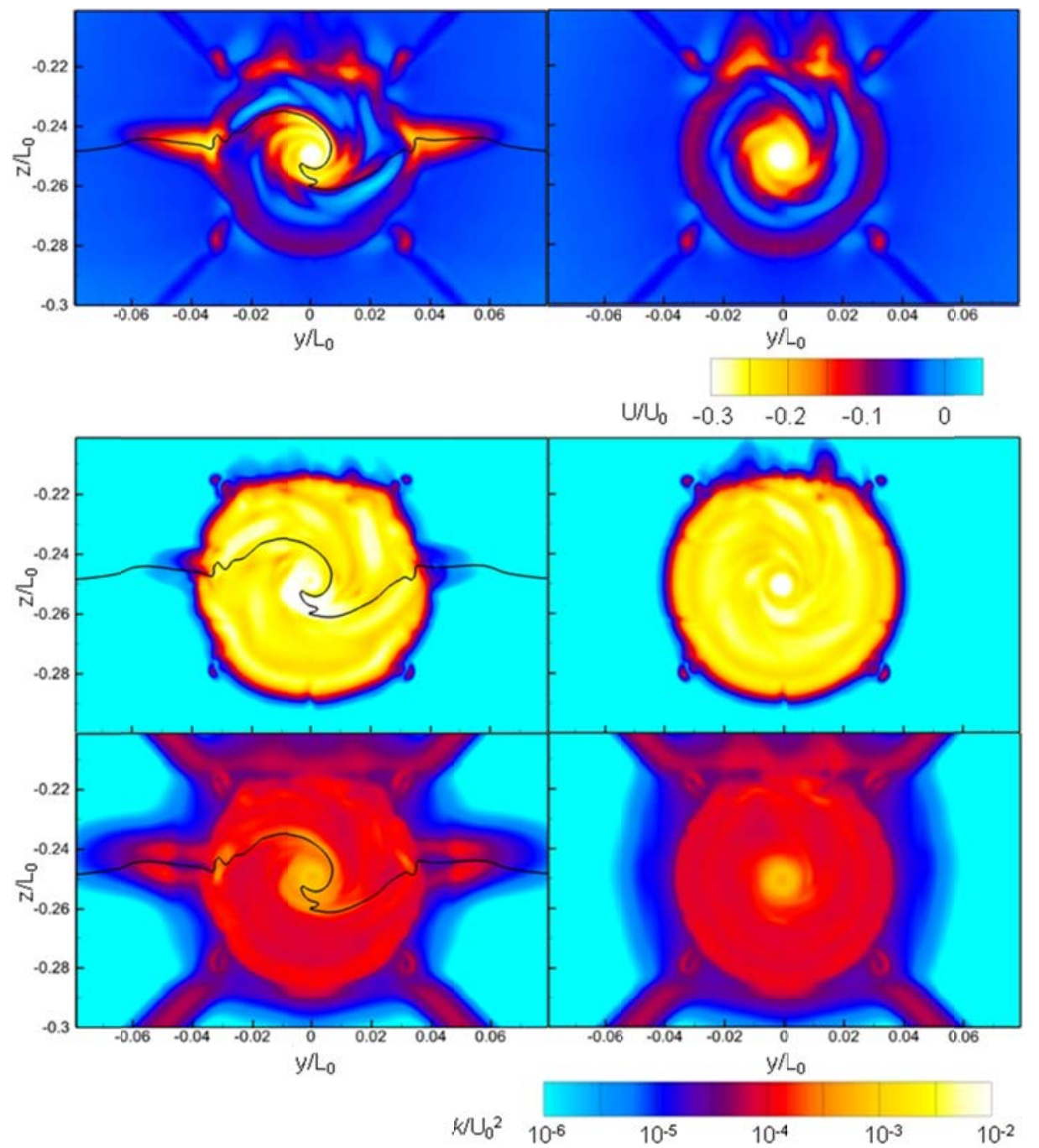

Figure 17: Mean velocity field 0.12 ship lengths downstream from the propeller for the selfpropelled Joubert BB2. Left column: stratified case with the pycnocline at the propeller shaft; right column: non-stratified case. The top panels show mean longitudinal velocity; center panels show resolved turbulent kinetic energy; bottom panels show modeled turbulent kinetic energy. The position of the $\rho=\rho_{0}+\Delta \rho / 2$ contour is shown as a solid black line. 


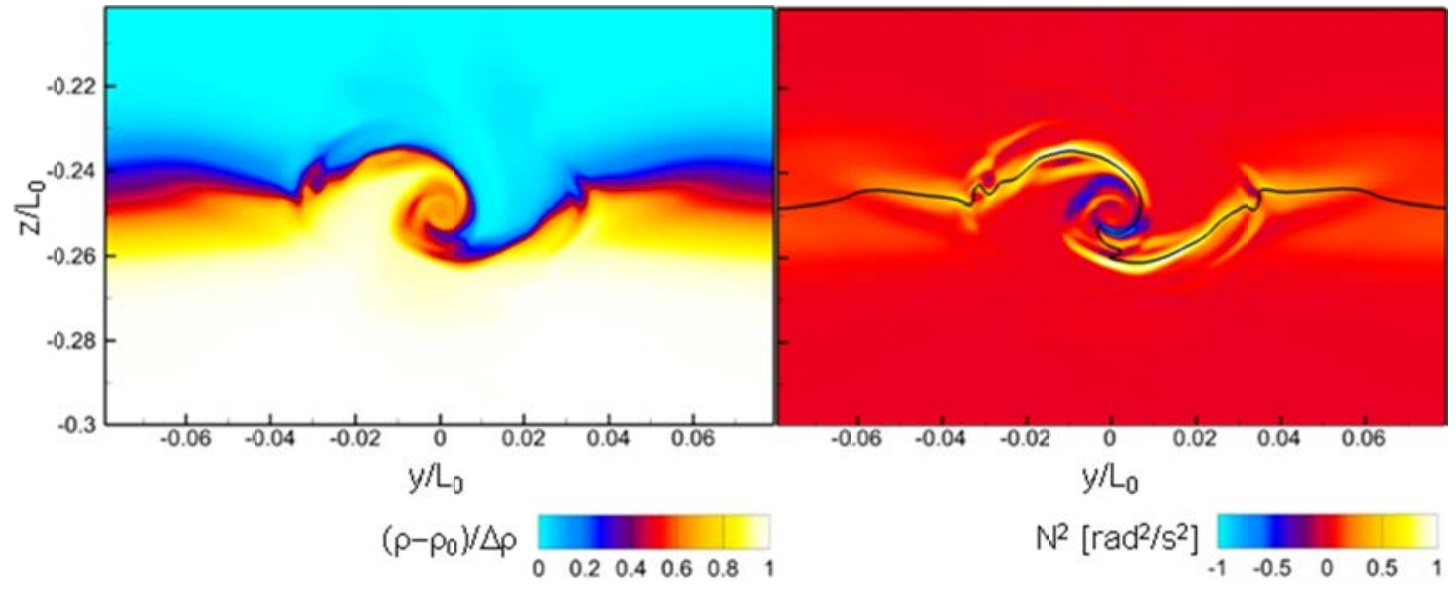

Figure 18: Density field 0.12 ship lengths downstream from the propeller for the self-propelled Joubert BB2, for stratified case with the pycnocline at the propeller shaft. Left: mean density; right: mean vertical density gradient, expressed as buoyancy frequency. 

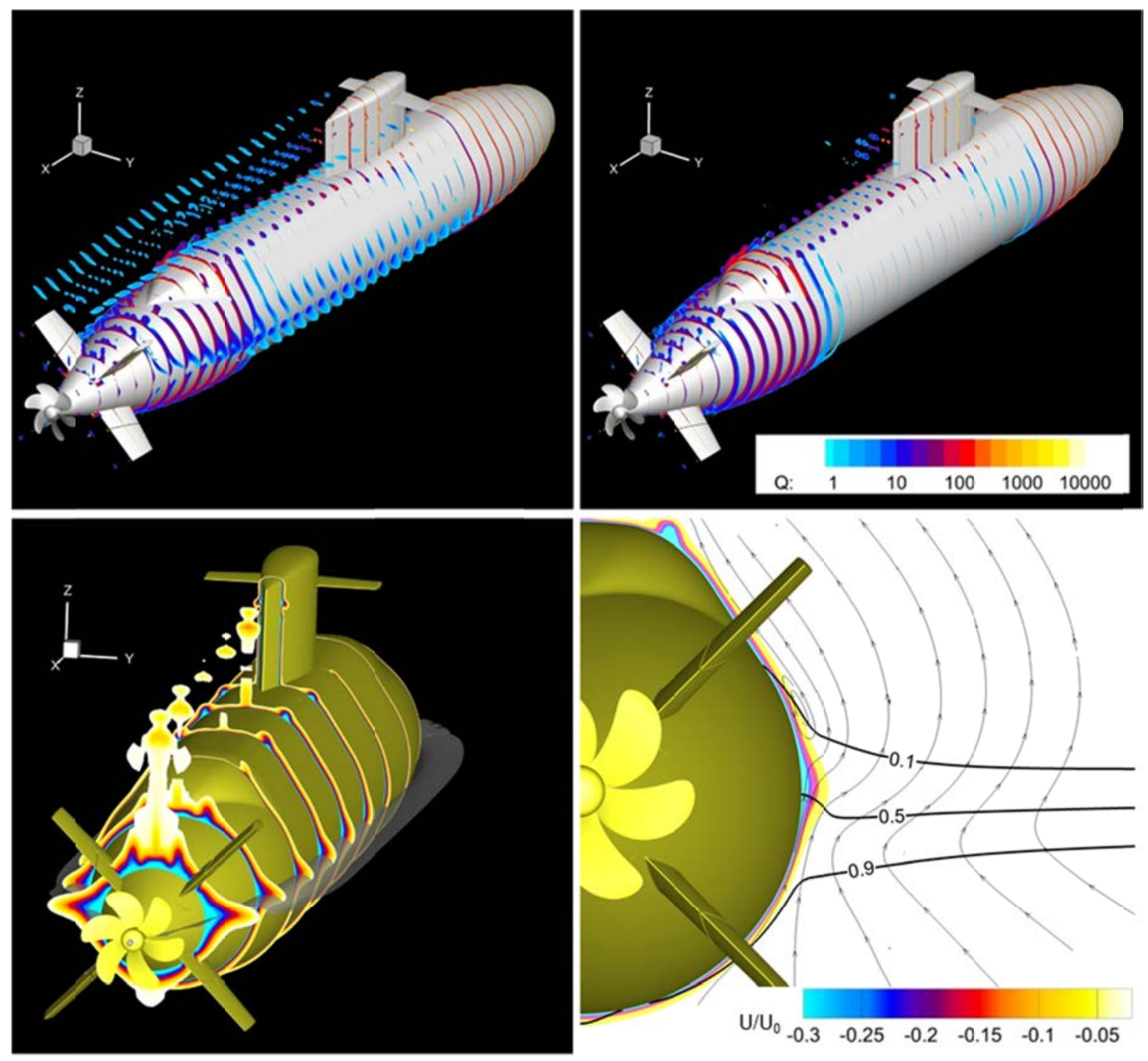

Figure 19: Top left: mean Q in the boundary layer of the Joubert BB2 submarine for the stratified case with the interface at the propeller shaft; top right: same for non-stratified case. Bottom left: mean longitudinal velocity on axial cross sections showing the boundary layer; bottom right: boundary layer and transverse streamlines in the mid-ships plane; three isosurfaces of mean density are also shown. 


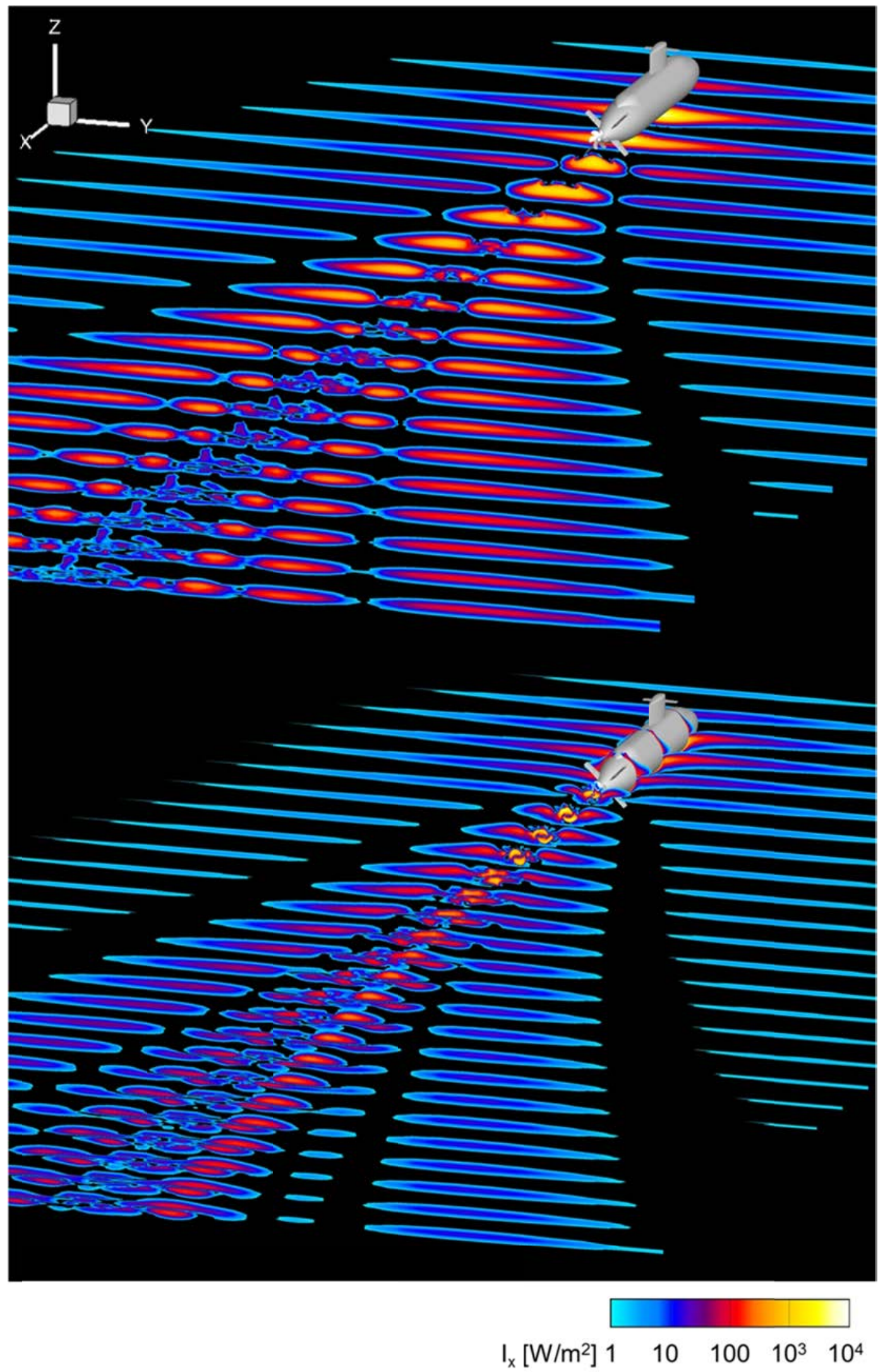

Figure 20. Potential power flux $I_{x}$ as defined by Eq. (10), for the self-propelled Joubert BB2. Top: pycnocline at $z_{0}=-0.33 L_{0}$; bottom: pycnocline at $z_{0}=-0.25 L_{0}$. 